\title{
Inclusive-jet and dijet production in polarized deep inelastic scattering
}

\author{
Ignacio Borsa $\odot^{*}$ \\ Departamento de Física and IFIBA, Facultad de Ciencias Exactas y Naturales, \\ Universidad de Buenos Aires, Ciudad Universitaria, Pabellón 1 (1428) Buenos Aires, Argentina \\ Daniel de Florian ${ }^{\dagger}$ and Iván Pedron $\odot^{\ddagger}$ \\ International Center for Advanced Studies (ICAS), ICIFI and ECyT-UNSAM, \\ 25 de Mayo y Francia, (1650) Buenos Aires, Argentina
}

(Received 28 October 2020; accepted 17 December 2020; published 8 January 2021)

\begin{abstract}
We present the calculation for single-inclusive jet production in (longitudinally) polarized deep-inelastic lepton-nucleon scattering at next-to-next-to leading order (NNLO) accuracy, based on the projection-toBorn method. As a necessary ingredient to achieve the NNLO results, we also introduce the next-toleading-order (NLO) calculation for the production of dijets in polarized DIS. Our dijet calculation is based on an extension of the dipole subtraction method to account for polarized initial-state partons. We analyze the phenomenological consequences of higher order QCD corrections for the Electron-Ion Collider kinematics.
\end{abstract}

DOI: 10.1103/PhysRevD.103.014008

\section{INTRODUCTION}

Much progress has been made in our understanding of the structure of hadrons over the last decades, both from the theoretical and the experimental sides. The study of the spin structure of hadrons in terms of its components, particularly the proton, is, however, still one of the challenges faced by particle physics. The spin content of the proton can be codified in terms of the polarized parton distributions of quarks and gluons, which can be experimentally probed in high energy collisions processes with polarized nucleons. Contrary to the case of unpolarized parton distributions, which have been extensively studied for a wide kinematical range, based on several complementary measurements from different observables, our knowledge on the helicity distributions for partons inside the proton is more limited. While more than 30 years ago, fixed-target deep-inelastic scattering (DIS) measurements from EMC refuted the naive interpretation of the parton model, proving that the amount of spin carried by quarks and antiquarks is relatively small [1], the exact decomposition of the proton spin between quarks, gluons and orbital angular momentum is still unclear. Polarized proton-proton collisions performed at

\footnotetext{
*iborsa@df.uba.ar

†deflo@unsam.edu.ar

*ipedron@unsam.edu.ar
}

Published by the American Physical Society under the terms of the Creative Commons Attribution 4.0 International license. Further distribution of this work must maintain attribution to the author(s) and the published article's title, journal citation, and DOI. Funded by SCOAP ${ }^{3}$. the BNL Relativistic Heavy-Ion-Collider (RHIC) [2], which receive significant contributions from gluon-initiated processes, improved the description of the gluon spin distribution, showing that its contribution to the proton spin is not negligible [3], although providing constraints only for a reduced range of proton momentum fractions. Furthermore, the amount of spin carried by the sea quarks is also still an open question [4,5]. In that sense, the future U.S.-based Electron-Ion-Collider (EIC), allowing a much wider kinematical range, and reaching an unprecedented precision for polarized measurements [6], is expected to provide new insights on the spin decomposition of the proton in terms of its fundamental building blocks [7-9].

In addition to high-precision measurements for a wider range of momentum fractions, the improvement of our picture of the proton spin will require a consistent increase in the accuracy of the theoretical description of the observables to be measured. It is known that leading order (LO) perturbative calculations $\mathcal{O}\left(\alpha_{S}^{0}\right)$ in QCD only provide qualitative descriptions, since higher order corrections in the strong coupling constant are sizable. Although a remarkable effort to compute higher order corrections for unpolarized processes has taken place during the last 30 years, setting next-to-next-to-leading order (NNLO) as the standard for Large-Hadron-Collider (LHC) calculations and even reaching the following order for some processes, the picture for polarized calculations is not as developed. Polarized calculations in dimensional regularization necessarily involve dealing with extensions of the $\gamma_{5}$ matrix and Levi-Civita tensor to an arbitrary number of dimension, making the computation much more intricate than its 
unpolarized counterpart. Until recently, NNLO corrections for polarized processes were only obtained for completely inclusive Drell-Yan [10] and DIS [11], in addition to the helicity splitting functions [12-14]. More exclusive observables provide results that can be directly compared to experimental data, and could, in principle, be used to disentangle individual contributions associated to different partons. In particular, jet production in DIS is an extremely useful tool to probe the partonic densities, since it can give a stronger grip on the gluon distribution, while avoiding nonperturbative corrections associated to final-state hadronization. Developments in techniques for flavor and charge tagging in jet production could further improve the potential of jet measurements to disentangle individual flavor contributions in global analysis $[15,16]$.

Higher order corrections are not only necessary to improve the accuracy of the theoretical description. It is also important to check the stability of the perturbative series, that is, how these corrections affect the resulting cross sections and spin asymmetries, since only processes perturbatively well behaved can be used as good probes for parton distributions, and be utilized for its extraction. Furthermore, for the specific case of jet production, it is only at higher orders in QCD that jet structure is fully developed, allowing us to realistically match the theoretical description to the experimental data and the cuts imposed in the jet reconstruction.

In this paper we present the NLO calculation for dijet production in polarized and unpolarized lepton-nucleon DIS, based on an extension of the Catani-Seymour dipole subtraction method [17] to account for polarized initial particles. We analyze the structure of higher order corrections in the Electron-Ion-Collider kinematics, its perturbative stability and phenomenological implications. Through a detailed study of the polarized cross sections and asymmetries we also identify the most important partonic contributions for different kinematical regions. Additionally, we expand on our previous results [18] for single-exclusive jet production in DIS at NNLO, achieved by combining the aforementioned dijet result with the inclusive polarized NNLO DIS structure functions [11] through the application of the projection-toBorn (P2B) method [19]. We analyze the perturbative stability of the higher order corrections to the cross section and asymmetries, as well as the contributions from the different partons to the NNLO corrections. Both the NLO single- and dijet, as well as the NNLO single-jet calculations are implemented in our code POLDIS [20].

The remaining of the paper is organized as follow: in Sec. II we begin by defining the kinematics of both singleand dijet production in DIS. In Sec. III we detail both our extension of the dipole subtraction method for polarized QCD processes, and its use in the P2B method in order to achieve polarized jet production at NNLO. In Sec. IV we present the phenomenological results for inclusive NLO dijet production at the EIC in the Breit-frame, and in Sec. V we do the same for inclusive NNLO single-jet production in the laboratory frame. Finally, in Sec. VI we summarize our work and present our conclusions.

\section{JET PRODUCTION KINEMATICS}

We start considering the case of inclusive-single jet production in DIS. Specifically, we study the process

$$
e(k)+P(p) \rightarrow e\left(k^{\prime}\right)+\operatorname{jet}\left(p_{T}, \eta\right)+X,
$$

where $k$ and $p$ are the momenta of the incoming electron and proton, respectively, and $k^{\prime}$ is the momentum of the outgoing electron. We work in the laboratory frame (L), where single-jet production receives nonvanishing contributions already at $\mathcal{O}\left(\alpha_{S}^{0}\right)$. We only consider, for the time being, neutral-current processes mediated by the exchange of a virtual photon, with its momentum $q=k-k^{\prime}$ and virtuality $Q^{2}=-q^{2}$ fully determined by the electron kinematics. The inelasticity $y$ and Bjorken variable $x$ are then defined as usual by

$$
y=\frac{q \cdot p}{k \cdot p}, \quad x=\frac{Q^{2}}{2 p \cdot q} .
$$

In addition to the variables commonly used for fully inclusive DIS, more insight on the underlying partonic kinematics can be obtained through the study of the finalstate jet, which can be characterized in terms of its transverse momentum $p_{T}$ with respect to the beam, and its pseudorapidity $\eta$.

At higher orders in $\alpha_{S}$, the production of multiple finalstate jets becomes available. Dijet production can be better studied in the Breit frame (B), where there is no contribution of $\mathcal{O}\left(\alpha_{S}^{0}\right)$ to the production of transverse jets. Formally, the Breit frame is defined as the one that satisfies $2 x \vec{p}+\vec{q}=0$. Note that for the $\mathcal{O}\left(\alpha_{S}^{0}\right)$ process, this implies that the virtual photon and incoming quark collide head-on, completely reversing the momentum of the quark (hence the commonly used nickname brick-wall frame), as its represented schematically in Fig. 1. The first non-vanishing contribution is then obtained at $\mathcal{O}\left(\alpha_{S}\right)$, with two final-state partons with opposed transverse momentum.

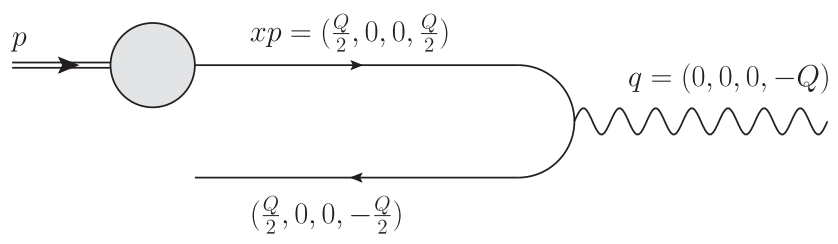

FIG. 1. $\quad \mathcal{O}\left(\alpha_{S}^{0}\right)$ Breit frame kinematics for the process $p_{1}(x p)+$ $\gamma^{*}(q) \rightarrow p_{2}(x p+q)$. 
For dijet production, we specify the process

$$
e(k)+P(p) \rightarrow e\left(k^{\prime}\right)+\operatorname{jet}\left(p_{T, 1}, \eta_{1}\right)+\operatorname{jet}\left(p_{T, 2}, \eta_{2}\right)+X .
$$

The availability of a second jet allows for a more indepth study of the partonic kinematics. As in the H1 [21,22] and ZEUS [23] experiments, and in addition to the jets transverse momentum and pseudorapidities, the dijet production cross section can be studied in term of the dijet variables such as the invariant mass $M_{12}$, the dijet momentum fraction $\xi_{2}$, as well as the average momentum $\left\langle p_{T}\right\rangle_{2}$ and pseudorapidity difference $\eta^{*}$ in the Breit-Frame, which are defined by

$$
\begin{aligned}
M_{12} & =\sqrt{\left(p_{1}+p_{2}\right)^{2}}, \\
\left\langle p_{T}\right\rangle_{2} & =\frac{1}{2}\left(p_{T, 1}^{B}+p_{T, 2}^{B}\right), \\
\eta^{*} & =\left|\eta_{1}^{B}-\eta_{2}^{B}\right|, \\
\xi_{2} & =x\left(1+\frac{M_{12}^{2}}{Q^{2}}\right) .
\end{aligned}
$$

It is worth noticing that, at the $\mathrm{LO}$ of dijet production, $\xi_{2}$ is the momentum fraction carried by the incoming parton.

\section{CALCULATION OF HIGHER ORDER CORRECTIONS}

Calculations beyond the leading order in QCD necessarily involves cancellations between the individually divergent pieces coming from infrared real emission and virtual diagrams, in addition to the factorization contributions. In the dimensional regularization scheme the number of dimensions is set to $D=4-2 \epsilon$, and those divergences then appear as poles in $\epsilon$. The cancellation between those poles can only be achieved after the integration of each of the divergent parts over its appropriate phase space, thus impeding a direct numerical calculation.

Several methods to numerically compute higher order corrections were developed over the last three decades. The two main approaches are based on either limiting the phase space integration (phase space slicing) in order to avoid the divergent regions, or generating appropriate counterterms (subtraction) to cancel the singularities in each of the pieces of the calculation. For the latter, the proposed counterterm should have the same divergent behavior as the real and collinear parts, while being simple enough to be integrated analytically in order to cancel the poles coming from the virtual diagrams.

Many general methods for constructing NLO counterterms were proposed. Among them, the dipole subtraction method developed by Catani and Seymour, and based on the dipole factorization formula, allows to calculate any jet production cross section at NLO accuracy. The landscape for the following order is complicated due to the appearance of many more singular configurations, but several methods of varying generality are also available for the computation of NNLO calculations [19,24-31]. In particular, for processes where the Born kinematic can be inferred from external non-QCD particles, the P2B method [19] allows to obtain $\mathrm{N}^{k} \mathrm{LO}$ differential calculations for a jet observable $\mathcal{O}$, given that the $\mathrm{N}^{k} \mathrm{LO}$ inclusive cross section and the differential $\mathrm{N}^{k-1} \mathrm{LO}$ for $\mathcal{O}+$ jet are known. Consequently, given a NLO dijet production in polarized DIS calculation and the polarized NNLO structure functions, we can then compute the NNLO exclusive single jet cross section. A similar approach was used in unpolarized DIS for the calculation of the N3LO corrections to singlejet production [32], using the previous NNLO dijet production results based on the antenna subtraction formalism [33] and the N3LO DIS structure functions [34].

This exclusive NNLO single-jet calculation is implemented in our code POLDIS for both polarized and unpolarized DIS. It allows to compute any infrared safe observable related to single-jet production at NNLO accuracy in the laboratory, as well as to single- and dijet production in the Breit-frame with NLO precision. The code is partially based on DISENT, which implements the Catani-Seymour dipole subtraction method to obtain the NLO single- and dijet cross sections in unpolarized DIS. Mayor modifications were made in order to include the polarized dijet computation, using an extended version of the dipole subtraction to account for initial-state polarized particles, as well as the implementation of the P2B subtraction in order to obtain NNLO results. Note that the previously reported bug in DISENT in the gluon channel [35-38] was fixed along with the modifications (see Appendix A).

Both the extension of the dipole subtraction as well as the P2B method will be discussed in more detail in Secs. III A and III B.

\section{A. The dipole subtraction method for polarized processes}

For processes involving (polarized) unpolarized initialstate hadrons, QCD calculations necessarily involve convolutions between partonic cross sections and (helicity) parton distribution functions, (p)PDFs, codifying the (spin) momentum distribution of partons inside that hadron. In the case of DIS scattering, the (polarized) unpolarized hadronic cross sections can be written perturbatively as:

$$
\begin{aligned}
(\Delta) \sigma(p)= & \sum_{a} \int_{0}^{1} d z(\Delta) f_{a}\left(z, \mu_{F}^{2}\right)\left[(\Delta) \hat{\sigma}_{a}^{\mathrm{LO}}(z p)\right. \\
& \left.+(\Delta) \hat{\sigma}_{a}^{\mathrm{NLO}}\left(z p, \mu_{F}^{2}\right)+\cdots\right]
\end{aligned}
$$

where ... denotes higher order corrections. The helicity pPDF for a parton $a$ carrying a fraction $z$ of the proton's momentum $p$ is defined as $\Delta f_{a}\left(z, \mu_{F}^{2}\right) \equiv f^{+}(z)-f^{-}(z)$, 
with $f^{+}(z)\left(f^{-}(z)\right)$ denoting the density of partons of type $a$ and momentum fraction $z$, with their helicities aligned (anti-aligned) with that of the proton. On the other hand, the polarized partonic cross section $\Delta \hat{\sigma} \equiv \frac{1}{2}\left[\hat{\sigma}^{++}-\hat{\sigma}^{+-}\right]$is defined in terms of the difference between the cross sections with the incoming lepton and hadron polarized parallel and antiparallel. Up to NLO, the (polarized) unpolarized $m$-parton cross section is given by

$$
\begin{gathered}
(\Delta) \hat{\sigma}_{a}^{\mathrm{LO}}(p)=\int_{m} d(\Delta) \hat{\sigma}_{a}^{B}(p), \\
(\Delta) \hat{\sigma}_{a}^{\mathrm{NLO}}\left(p, \mu_{F}^{2}\right)=\int_{m+1} d(\Delta) \hat{\sigma}_{a}^{R}(p)+\int_{m} d(\Delta) \hat{\sigma}_{a}^{V}(p) \\
+\int_{m} d(\Delta) \hat{\sigma}_{a}^{C}(p),
\end{gathered}
$$

where $d(\Delta) \hat{\sigma}^{B}$ is the (polarized) unpolarized partonic Born cross section, and $d(\Delta) \hat{\sigma}^{R}$ and $d(\Delta) \hat{\sigma}^{V}$ stand for the NLO partonic real-emission and virtual matrix elements, respectively. The last term in Eq. (5) is associated to the collinear factorization that must be introduced in the case of cross sections involving initial hadrons, to account for the divergences arising from initial-state radiation.

It is worth noticing that we are working in $D=4-2 \epsilon$ dimensions, and that each of the integrals in Eq. (5) is separately divergent in the limit $\epsilon \rightarrow 0$. The calculation of polarized cross sections in dimensional regularization is more involved than its unpolarized counterpart, since the extension of the $\gamma_{5}$ matrix and the Levi-Civita tensor $\epsilon^{\mu \nu \sigma \rho}$ appearing in the helicity projection operators in $D$ dimensions is far from trivial. One way to consistently treat $\gamma_{5}$ and $\epsilon^{\mu \nu \sigma \rho}$ is in the HVBM ('t Hooft-Veltman-BreitenlohnerMaison) scheme [39,40], in which the $D$-dimensional space is separated in the standard four-dimensional subspace, and a $(D-4)$-dimensional subspace. In this scheme, $\epsilon^{\mu \nu \sigma \rho}$ is treated as a genuinely four-dimensional tensor, while $\gamma_{5}$ is such that $\left\{\gamma_{5}, \gamma^{\mu}\right\}=0$ for $\mu=0,1,2,3$, and $\left[\gamma_{5}, \gamma^{\mu}\right]=0$ otherwise.

An alternative to numerically compute the partonic cross section in Eq. (5) is the so-called dipole subtraction method, introduced by Catani and Seymour [17] as a general framework for the calculation of NLO jet cross sections. This is the method used to compute the NLO corrections of jet observables in both DISENT and POLDIS. As in other subtraction-based approaches, the idea behind the procedure is to cancel the infrared singularities that appear in the real, virtual and collinear-factorization pieces of the (polarized) unpolarized cross section, which are integrated in different phase spaces $(m$ particles for the virtual diagrams and $m+1$ for the real-emission diagrams), already at the integral level. That cancellation of divergences is achieved through the introduction of a counterterm $d(\Delta) \sigma^{A}$ that has the same infrared behavior (in $D$ dimensions) as $d(\Delta) \sigma^{R}$. By adding and subtracting this term, the NLO calculation can be rewritten as

$$
\begin{aligned}
\int d(\Delta) \hat{\sigma}^{\mathrm{NLO}}= & \int_{m+1}\left(d(\Delta) \hat{\sigma}^{R}-d(\Delta) \hat{\sigma}^{A}\right) \\
& +\int_{m}\left(d(\Delta) \hat{\sigma}^{V}+d(\Delta) \hat{\sigma}^{C}+\int_{1} d(\Delta) \hat{\sigma}^{A}\right) .
\end{aligned}
$$

In Eq. (6) the first integral can be numerically performed in four-dimensions since $d(\Delta) \sigma^{A}$ acts as a local counterterm of $d(\Delta) \sigma^{R}$. In the second term the cancellation of $\epsilon$ poles requires the integrals to be performed analytically.

Clearly, the key of the subtraction method lies in the construction of $d(\Delta) \sigma^{A}$, which in addition to reproduce the divergent behavior of $d(\Delta) \sigma^{R}$ should be simple enough to be analytically integrated. In this case the term is constructed by the use of the dipole factorization formula

$$
d(\Delta) \hat{\sigma}^{A}=\sum_{\text {dipoles }} d(\Delta) \hat{\sigma}^{B} \otimes d(\Delta) V_{\text {dipole }}
$$

in the collinear and soft limits, where $\otimes$ stands for the appropriate phase space convolution and sums over color and spin indices. The $(\Delta) V_{\text {dipole }}$ are the universal dipole factors that match the infrared singular behavior of $d(\Delta) \sigma^{R}$. Note that these terms need to be analytically integrable if $D$-dimensions over the single-parton spaces related to soft and collinear divergences in order to make use Eq. (6). The construction of these dipole factors for the unpolarized case was already outlined in detail in Catani and Seymour's paper. We now discuss the extension to the particular case of cross sections with one initial-state polarized parton, required for the calculation of the polarized DIS process.

Following the same notation introduced by Catani and Seymour, the complete polarized local counterterm $d \Delta \hat{\sigma}_{a}^{A}$ is

$$
\begin{aligned}
d \Delta \hat{\sigma}_{a}^{A}= & \mathcal{N}_{\text {in }} \frac{1}{n_{c}(a) \Phi\left(p_{a}\right)} \sum_{\{m+1\}} d \phi_{m+1}\left(p_{1}, \ldots, p_{m+1} ; p_{a}\right) \\
& \times \frac{1}{S_{\{m+1\}}}\left\{\sum_{\text {pairs } i, j} \sum_{k \neq i, j} \Delta \mathcal{D}_{i j, k}\left(p_{1}, \ldots, p_{m+1} ; p_{a}\right)\right. \\
& +\sum_{\text {pairs } i, j} \Delta \mathcal{D}_{i j}^{a}\left(p_{1}, \ldots, p_{m+1} ; p_{a}\right) \\
& \left.+\sum_{i} \sum_{k \neq i} \Delta \mathcal{D}_{k}^{a i}\left(p_{1}, \ldots, p_{m+1} ; p_{a}\right)\right\}
\end{aligned}
$$

where the terms $\Delta \mathcal{D}_{i j, k}, \Delta \mathcal{D}_{i j}^{a}$ and $\Delta \mathcal{D}_{k}^{a i}$ represent the dipole subtraction terms for final-state singularities with a final-state spectator, final-state singularities with an initialstate spectator, and initial-state singularities, respectively. The sum is performed over all the possible $m+1$ final-state partons configurations, with $d \phi_{m+1}$ denoting the corresponding phase space. Additionally, the $1 / n_{c}$ accounts for the average over the initial-state colors, $\Phi\left(p_{a}\right)$ is flux 
factor, and $S_{\{m\}}$ is the Bose symmetry factor for identical particles in the final-state. In $\mathcal{N}_{\text {in }}$ the rest of the QCDindependent factors are included.

It is important to note that to create local counter-terms for the polarized DIS NLO cross section, only the polarization of the initial-state parton needs to be considered. In this case, instead of taking the average of its polarizations, the difference between them is used. Spin states of finalstate parton are summed over and therefore they are treated as unpolarized. Thus, the dipole subtraction terms $\Delta \mathcal{D}_{i j, k}$ and $\Delta \mathcal{D}_{i j}^{a}$, associated to final-state singularities, are constructed as in ref. [17] (using the corresponding polarized Born cross section). New expressions for the dipole formulas are therefore only needed in the case of initialstate singularities with one initial-state parton, represented by $\Delta \mathcal{D}_{k}^{a i}$.

As in the case of the unpolarized cross sections, the terms $\Delta \mathcal{D}_{k}^{a i}$ can be obtained from the dipole factorization formula. In the limit $p_{a} \cdot p_{i} \rightarrow 0$, where $p_{a}$ is the moment of the initial-state parton and $p_{i}$ a final-state one, the dipole factorization formula for the polarized $(m+1)$-parton matrix element can be expressed as

$$
\begin{gathered}
{ }_{m+1, a}\langle 1, \ldots, m+1 ; \Delta a \| 1, \ldots, m+1 ; \Delta a\rangle_{m+1, a} \\
=\sum_{k \neq i} \Delta \mathcal{D}_{k}^{a i}\left(p_{1}, \ldots, p_{m+1} ; p_{a}\right)+\cdots
\end{gathered}
$$

where $|1, \ldots, m ; \Delta a\rangle_{m, a}$ represents an $m$-particle state in the color and helicity space, with $\Delta a$ denoting that the difference between the incoming parton polarizations is considered. The $\sum_{k} \Delta \mathcal{D}_{k}^{a i}$ stands for the sum of the polarized dipole contributions, in which the partons $a$ and $i$ act as a single initial-state parton $\widetilde{a}$, the "emitter," and the finalstate parton $k$ acts as the "spectator" $\tilde{k}$. The $\ldots$ stands for the other nonsingular terms in the $p_{a} \cdot p_{i} \rightarrow 0$ limit. Each dipole contribution is given by

$$
\begin{aligned}
\Delta \mathcal{D}_{k}^{a i}( & \left.p_{1}, \ldots, p_{m+1} ; p_{a}\right) \\
= & -\frac{1}{2 p_{a} \cdot p_{i}} \frac{1}{x_{i k, a}} \cdot{ }_{m, a}\langle 1, \ldots, \tilde{k}, \ldots, m+1 ; \Delta \tilde{a} i| \\
& \times \frac{\boldsymbol{T}_{k} \cdot \boldsymbol{T}_{a i}}{\boldsymbol{T}_{a i}^{2}} \Delta \boldsymbol{V}_{k}^{a i}|1, \ldots, \tilde{k}, \ldots, m+1 ; \Delta \tilde{a} i\rangle_{m, a} .
\end{aligned}
$$

The $\boldsymbol{T}$ are the color charge operators corresponding to each parton. The emitter and spectator momenta are given respectively by $\tilde{p}_{a i}^{\mu}=x_{i k, a} p_{a}^{\mu}$ and $\tilde{p}_{k}^{\mu}=p_{k}^{\mu}+p_{i}^{\mu}-$ $\left(1-x_{i k, a}\right) p_{a}^{\mu}$, where

$$
x_{i k, a}=\frac{p_{k} p_{a}+p_{i} p_{a}-p_{i} p_{k}}{p_{k} p_{a}+p_{i} p_{a}} .
$$

The splitting functions $\Delta \boldsymbol{V}_{k}^{a i}$ are the only new blocks needed for the extension of the dipole subtraction formalism to the polarized case. They are constructed so that they give the correct eikonal factors in the soft limits, and the correct $D$-dimensional polarized Altarelli-Parisi splitting functions $\Delta P_{i j}$ in the corresponding collinear limits. Similarly to $\Delta P_{i j}, \Delta \boldsymbol{V}_{k}^{a i}$ are matrices in the helicity space of the emitter parton $\tilde{a} i$, and their expression in given by:

$$
\begin{gathered}
\left\langle s\left|\Delta \boldsymbol{V}_{k}^{q_{a} g_{i}}\right| s^{\prime}\right\rangle=8 \pi \mu^{2 \epsilon} \alpha_{s} C_{F}\left[\frac{2}{1-x_{i k, a}+u_{i}}-\left(1+x_{i k, a}\right)+3 \epsilon\left(1-x_{i k, a}\right)\right] \delta_{s s^{\prime}}, \\
\left\langle s\left|\Delta \boldsymbol{V}_{k}^{g_{a} \bar{q}_{i}}\right| s^{\prime}\right\rangle=8 \pi \mu^{2 \epsilon} \alpha_{s} T_{R}\left[2 x_{i k, a}-1-2 \epsilon\left(1-x_{i k, a}\right)\right] \delta_{s s^{\prime}}, \\
\left\langle\mu\left|\Delta \boldsymbol{V}_{k}^{q_{a} q_{i}}\right| \nu\right\rangle=8 \pi \mu^{2 \epsilon} \alpha_{s} C_{F}\left\{i \frac{\left(1-u_{i}\right)}{p_{a} p_{k}}\left[x_{i k, a}+2\left(1-x_{i k, a}\right)(1+\epsilon)\right] \epsilon^{\alpha \beta \mu \nu}\left(p_{i}^{\alpha}+p_{k}^{\alpha}\right) p_{a}^{\beta}\right\}, \\
\left\langle\mu\left|\Delta \boldsymbol{V}_{k}^{g_{a} g_{i}}\right| \nu\right\rangle=16 \pi \mu^{2 \epsilon} \alpha_{s} C_{A}\left\{i \frac{\left(1-u_{i}\right)}{p_{a} p_{k}}\left[\frac{1}{1-x_{i k, a}+u_{i}}-1+2\left(1-x_{i k, a}\right)(1+\epsilon)\right] \epsilon^{\alpha \beta \mu \nu}\left(p_{i}^{\alpha}+p_{k}^{\alpha}\right) p_{a}^{\beta}\right\},
\end{gathered}
$$

where $u_{i}=p_{a} p_{i} /\left(p_{a} p_{i}+p_{a} p_{k}\right)$.

Notice, however, that these expressions of the splitting functions $\Delta \boldsymbol{V}_{k}^{a i}$ as matrices in the helicity states of $\tilde{a} i$ are not really needed in the polarized case since the spin structure is trivial for both quarks and gluons. This is due to the fact that the spin correlation terms cancel out due to parity conservation in polarized processes (See
Appendix B). Therefore, only the difference between the possible spin states of the emitter parton $\tilde{a} i$ are required to perform the subtraction. In the case of a gluon emitter, this accounts for the contraction with the tensor $i \epsilon^{\rho \sigma \mu \nu} p_{a}^{\rho} n^{\sigma} /\left(2 p_{a} \cdot n\right)$, where $n$ is any lightlike vector that satisfies $n \cdot p_{a} \neq 0$, while for a quark emitter the tensor $\delta^{s s^{\prime}} / 2$ is used. The resulting kernels are 
$\frac{\Delta \boldsymbol{V}_{k}^{q_{a} g_{i}}(x ; u)}{8 \pi \alpha_{s} \mu^{2 \epsilon}}=C_{F}\left[\frac{2}{1-x+u}-(1+x)+3 \epsilon(1-x)\right]$,

$$
\begin{gathered}
\frac{\Delta \boldsymbol{V}_{k}^{g_{a} \bar{q}_{i}}(x)}{8 \pi \alpha_{s} \mu^{2 \epsilon}}=T_{R}[2 x-1-2 \epsilon(1-x)], \\
\frac{\Delta \boldsymbol{V}_{k}^{q_{a} q_{i}}(x)}{8 \pi \alpha_{s} \mu^{2 \epsilon}}=C_{F}[x+2(1-x)(1+\epsilon)], \\
\frac{\Delta \boldsymbol{V}_{k}^{g_{a} g_{i}}(x ; u)}{8 \pi \alpha_{s} \mu^{2 \epsilon}}=2 C_{A}\left[\frac{1}{1-x+u}-1+2(1-x)(1+\epsilon)\right] .
\end{gathered}
$$

In order to integrate the dipole subtraction term, $\int_{m+1} d \sigma^{A}$, the $D$-dimensional integrals of the $\Delta \boldsymbol{V}_{k}^{a i}$ terms over the dipole phase space $d p_{i}\left(\tilde{p_{k}} ; p_{a}, x\right)$ are needed. The

procedure to obtain them is the same one outlined by Catani and Seymour. The resulting expressions $\Delta \mathcal{V}^{a, a i}$ are

$$
\begin{aligned}
\Delta \mathcal{V}^{q g}(x ; \epsilon)= & -\frac{1}{\epsilon} \Delta P^{g q}(x, 0)+\Delta P^{g q}(x, 0) \ln (1-x) \\
& -2 C_{F}(1-x)+\mathcal{O}(\epsilon), \\
\Delta \mathcal{V}^{g q}(x ; \epsilon)= & -\frac{1}{\epsilon} \Delta P^{q g}(x, 0)+\Delta P^{q g}(x, 0) \ln (1-x) \\
& +2 T_{R}(1-x)+\mathcal{O}(\epsilon), \\
\Delta \mathcal{V}^{q q}(x ; \epsilon)= & -\frac{1}{\epsilon} \Delta P^{q q}(x, 0)+\delta(1-x) C_{F}\left[\frac{1}{\epsilon^{2}}+\frac{3}{2 \epsilon}+\frac{\pi^{2}}{6}\right] \\
& +C_{F}\left[-\left(\frac{4}{1-x} \ln \frac{1}{1-x}\right)_{+}-\frac{2}{1-x} \ln (2-x)\right. \\
& -3(1-x)-(1+x) \ln (1-x)]+\mathcal{O}(\epsilon)
\end{aligned}
$$

where $x$ is the phase space convolution variable and the $\Delta P^{a b}(x, 0)$ are the aforementioned polarized four-dimensional Altarelli-Parisi kernels. In the HVBM scheme they are given by [41]:

$$
\begin{gathered}
\Delta P^{q q}(x, \epsilon)=C_{F}\left[\frac{2}{(1-x)_{+}}-(1+x)+3 \epsilon(1-x)\right. \\
\left.+\frac{3+\epsilon}{2} \delta(1-x)\right], \\
\Delta P^{q g}(x, \epsilon)=T_{R}[2 x-1-2 \epsilon(1-x)], \\
\Delta P^{g q}(x, \epsilon)=C_{F}[2-x+2 \epsilon(1-x)], \\
\Delta P^{g g}(x, \epsilon)=2 C_{A}\left[\frac{1}{(1-x)_{+}}-2 x+1+2 \epsilon(1-x)\right] \\
+\left(\frac{\beta_{0}}{2}+\frac{C_{A}}{6} \epsilon\right) \delta(1-x) .
\end{gathered}
$$

A final remark must be made about the polarized factorization counterterms $d \Delta \hat{\sigma}_{a}^{C}$ in Eq. (5). These counterterms are explicitly written as:

$$
\begin{aligned}
d \Delta \hat{\sigma}_{a}^{C}= & -\frac{\alpha_{S}}{2 \pi} \frac{1}{\Gamma(1-\epsilon)} \sum_{b} \int_{0}^{1} d z\left[-\frac{1}{\epsilon}\left(\frac{4 \pi \mu^{2}}{\mu_{F}^{2}}\right)^{\epsilon} \Delta P^{a b}(z, \epsilon)\right. \\
& \left.+\Delta K_{F . S}^{a b}\right] d \sigma_{b}^{B}(z p)
\end{aligned}
$$

where the value of $\Delta K_{F . S}^{a b}$ determines the factorization scheme. We work in the conventional polarized $\overline{\mathrm{MS}}$ factorization scheme in which one needs to compensate for the difference between the polarized and unpolarized quark splitting functions $\left(\Delta P^{q q}(x, \epsilon)\right.$ and $P^{q q}(x, \epsilon)$, respectively) in $D$-dimensions. Since the difference between the two kernels is given by $\Delta P^{q q}(x, \epsilon)-P^{q q}(x, \epsilon)=$ $4 \epsilon C_{F}(1-x)$, this is equivalent to setting $\Delta K_{F . S}^{q q}=$ $4 C_{F}(1-x)$ and $\Delta K_{F . S}^{a b}=0$ otherwise in Eq. (28).

\section{B. The projection-to-Born method}

As it was mentioned, the P2B method allows to obtain the $\mathrm{N}^{k} \mathrm{LO}$ calculation for a differential observable, provided that its inclusive cross section at that order, as well as the differential cross section for the observable plus a jet are known at $\mathrm{N}^{k-1} \mathrm{LO}$. The idea behind the method is to cancel the most divergent parts by simply using the full matrix 
element at each phase space point as a counterterm, but binning it in an equivalent Born-projected kinematics of the leading order process (hence the name "projection-toBorn"). That is, for each event with weight $w$, a counterterm with weight $-w$ is generated, but with the measurement function evaluated in the kinematics of an equivalent leading order process. Note that this requires the Born kinematics to be fully determined by external non-QCD particles.

The differential cross section for an observable $\mathcal{O}$ at $\mathrm{N}^{k} \mathrm{LO}$ accuracy can be written as:

$$
d \sigma_{\mathcal{O}}^{\mathrm{N}^{k} \mathrm{LO}}=d \sigma_{\mathcal{O}+\mathrm{jet}}^{\mathrm{N}^{k-1} \mathrm{LO}}-d \sigma_{\mathcal{O}_{\mathrm{P} 2 \mathrm{~B}}+\mathrm{jet}}^{\mathrm{N}^{k-1} \mathrm{LO}}+d \sigma_{\mathcal{O} \text { incl }}^{\mathrm{N}^{k} \mathrm{LO}},
$$

where in $d \sigma_{\mathcal{O}+\text { jet }}^{\mathrm{N}^{k-1} \mathrm{LO}}$ infrared cancellation at the $\mathrm{N}^{k-1} \mathrm{LO}$ level has already taken place (numerical implementations beyond leading-order thus require the use of an additional subtraction method). It should be noted that as the finalstate partons approach the most singular regions, they are clustered in a jet configuration with Born kinematics, and thus the born-projected counterterms exactly cancel the divergent behavior of the cross section. The appearance of the inclusive term in Eq. (29) is due to the fact that the sum of all the projected events in a given Born phase space point is equivalent to the integration of the additional final-state partons associated with real emission. Thus, the combination of the Born-projected terms along with the $k$-loop virtual diagrams results in the full contribution of the inclusive $\mathrm{N}^{k} \mathrm{LO}$ cross section to the observable under consideration.
Clearly, the key of the P2B method lies in the kinematical mapping $\mathcal{O} \rightarrow \mathcal{O}_{\text {P2B }}$. In the Born level DIS kinematics the momenta of the incoming and outgoing partons are fully determined by the lepton kinematics. The incoming parton has momenta $p=x P$, and the outgoing one $p^{\prime}=x P+q$. So the mapping to the Born kinematics is given by using these parton momenta to evaluate the measurement function for the born-projected counterterms. Note that this mapping only works in jet production in the laboratory frame, since in the Breit-frame the first nonvanishing contributions starts at order $\mathcal{O}\left(\alpha_{s}\right)$, with two final-state partons (and hence no mapping is possible in terms of $P, x$, and $q$ ).

In the particular case of single jet production in unpolarized (polarized) DIS at NNLO, the corresponding counterterms are generated from the double-real and one-loop real radiation matrix elements. The combination of those counterterms with the two-loop matrix elements is then equal to the unpolarized (polarized) DIS inclusive cross section at NNLO [11,34,42]. As mentioned, a numerical implementation of the calculation has yet to deal with the subleading divergences coming from the single-real radiation and one-loop diagrams contributing to the unpolarized (polarized) dijet cross section at NLO. Those missing blocks can then be calculated with the implementation of the Catani-Seymour dipole formalism, whose extension to the polarized case was discussed in III A. We can then rewrite Eq. (29) for the production of jets in unpolarized (polarized) DIS at NNLO in terms of the counterterms of Eq. (6) as:

$$
\begin{aligned}
(\Delta) \hat{\sigma}_{\mathcal{O}}^{\mathrm{NNLO}}= & (\Delta) \hat{\sigma}_{\mathcal{O}+\mathrm{jet}}^{\mathrm{NLO}}-(\Delta) \hat{\sigma}_{\mathcal{O}_{\mathrm{P} 2 \mathrm{~B}}+\mathrm{jet}}^{\mathrm{NLO}}+(\Delta) \hat{\sigma}_{\mathcal{O} \text { incl }}^{\mathrm{NNLO}} \\
= & \int_{m+1}\left[d(\Delta) \hat{\sigma}_{\mathcal{O}+\mathrm{jet}}^{R}-d(\Delta) \hat{\sigma}_{\mathcal{O}+\mathrm{jet}}^{A}\right]+\int_{m}\left[d(\Delta) \hat{\sigma}_{\mathcal{O}+\mathrm{jet}}^{V}+d(\Delta) \hat{\sigma}_{\mathcal{O}+\mathrm{jet}}^{C}+\int_{1} d(\Delta) \hat{\sigma}_{\mathcal{O}+\mathrm{jet}}^{A}\right] \\
& -\int_{m+1}\left[d(\Delta) \hat{\sigma}_{\mathcal{O}_{\mathrm{P} 2 \mathrm{~B}}+\mathrm{jet}}^{R}-d(\Delta) \hat{\sigma}_{\mathcal{O}_{\mathrm{P} 2 \mathrm{~B}}+\mathrm{jet}}^{A}\right]-\int_{m}\left[d(\Delta) \hat{\sigma}_{\mathcal{O}_{\mathrm{P} 2 \mathrm{~B}}+\mathrm{jet}}+d(\Delta) \hat{\sigma}_{\mathcal{O}_{\mathrm{P} 2 \mathrm{~B}}+\mathrm{jet}}^{C}+\int_{1} d(\Delta) \hat{\sigma}_{\mathcal{O}_{\mathrm{P} 2 \mathrm{~B}}+\mathrm{jet}}^{A}\right]+(\Delta) \hat{\sigma}_{\mathcal{O} \text { incl }}^{\mathrm{NNLO}}
\end{aligned}
$$

where we have used that the inclusive part can be expressed in terms of the $\mathrm{P} 2 \mathrm{~B}$ counterterms and the double-virtual matrix element for the observable $\mathcal{O}$ as:

$$
\begin{aligned}
& (\Delta) \hat{\sigma}_{\mathcal{O} \text { incl }}^{\mathrm{NNLO}}=\int_{m+1} d(\Delta) \hat{\sigma}_{\mathcal{O}_{\mathrm{P} 2 \mathrm{~B}}+\mathrm{jet}} \\
& +\int_{m}\left[d(\Delta) \hat{\sigma}_{\mathcal{O}_{\mathrm{P} 2 \mathrm{~B}}+\mathrm{jet}}^{V}+d(\Delta) \hat{\sigma}_{\mathcal{O}_{\mathrm{P} 2 \mathrm{~B}}+\mathrm{jet}}\right] \\
& +\int_{m-1} d(\Delta) \hat{\sigma}_{\mathcal{O}}^{V V} .
\end{aligned}
$$

In addition, the complete expression for the counterterm $d(\Delta) \hat{\sigma}_{\mathcal{O}+\text { jet }}^{A}$ is that given by Eq. (8).

\section{RESULTS OF POLARIZED NLO DIJET PRODUCTION}

The first step to reach NNLO accuracy for jet production in DIS lies in the calculation of the NLO dijet cross section. Precisely, in this section we present our results for polarized inclusive dijet production at NLO in the Breit frame (B). We consider the Electron-Ion Collider kinematics, with beam energies of $E_{e}=18 \mathrm{GeV}$ and $E_{p}=275 \mathrm{GeV}$, and reconstruct the jets with the anti- $k_{T}$ algorithm and $E$-scheme recombination $(R=1)$. Furthermore, for dijet production we fix the normalization and factorization scales central values as $\mu_{F}^{2}=\mu_{R}^{2}=\frac{1}{2}\left(Q^{2}+\left\langle p_{T}^{B}\right\rangle_{2}^{2}\right) \equiv \mu_{0}^{2}$, 
with $\alpha_{s}$ evaluated at NLO accuracy with $\alpha_{s}\left(M_{z}\right)=0.118$, and require that the pair of leading jets satisfy the kinematical cuts:

$$
\begin{aligned}
p_{T, 1}^{B} & >5 \mathrm{GeV}, \\
p_{T, 2}^{B} & >4 \mathrm{GeV}, \\
\left|\eta^{L}\right| & <3.5,
\end{aligned}
$$

with the $\eta$ cut imposed in the laboratory frame, while the lepton kinematics is restricted by

$$
\begin{aligned}
0.2 & <y<0.6, \\
25 \mathrm{GeV}^{2} & <Q^{2}<2500 \mathrm{GeV}^{2} .
\end{aligned}
$$

The parton distributions sets used were the NLOPDF4LHC15 [43] and DSSV $[3,44]$ for the unpolarized and polarized case, respectively.
We begin by presenting the $\mathrm{LO}$ and NLO results for the unpolarized and polarized cross sections in Fig. 2, in terms of the leading and subleading jet transverse momentum, $p_{T, 1}^{B}$ and $p_{T, 2}^{B}$, respectively. The lower inset in each plot shows the corresponding $K$-factor, defined as the ratio to the LO cross section $\sigma_{\mathrm{LO}}$, in order to quantify the effect of the NLO corrections. The bands presented in Fig. 2 represent the estimation for the theoretical uncertainties, obtained by independently varying the renormalization and factorization scales as $\mu_{R}, \mu_{F}=[1 / 2,2] \mu_{0}$ (with the additional constrain $\left.1 / 2 \leq \mu_{F} / \mu_{R} \leq 2\right)$.

For all the distributions, rather large NLO corrections are obtained, particularly for the low $p_{T}^{B}$ bins. These sizable corrections are associated to the asymmetric cuts chosen for the $p_{T}^{B}$ of the leading and subleading jets, which can already be noted in the different coverage of each of the distributions. It is also worth noticing the difference in sign of the NLO corrections, which enhance the distributions of the leading jet, while suppressing the subleading jet
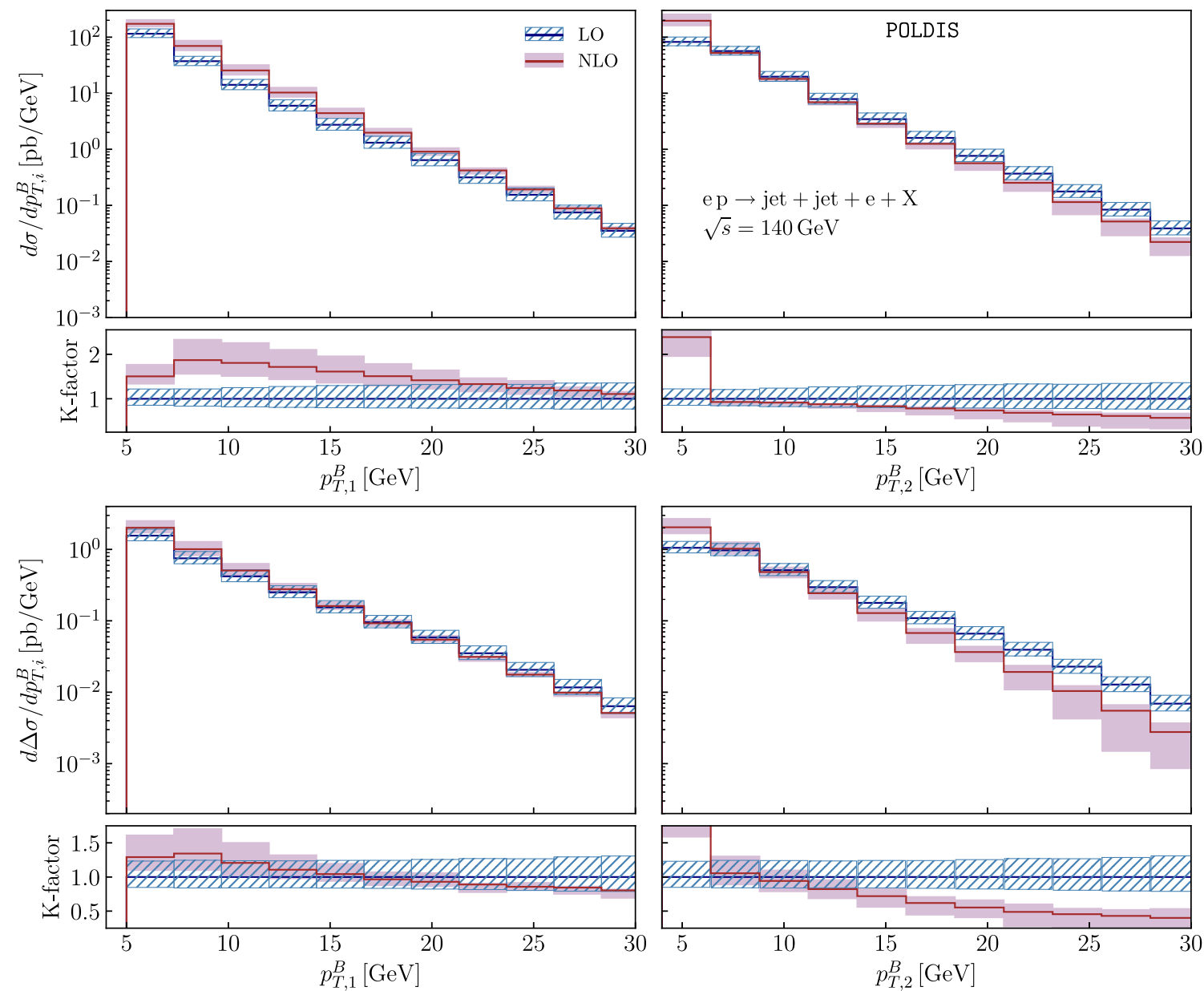

FIG. 2. Inclusive dijet production distributions as a function of the leading and sub-leading jet transverse momentum, $p_{T, 1}^{B}$ and $p_{T, 2}^{B}$, respectively, for both the polarized and unpolarized cases. The bands reflect the seven point variation in the cross section when independently changing the scales as $\mu_{R}, \mu_{F}=[1 / 2,2] \frac{1}{2} \mu_{0}$. The lower inset for each plot depicts the $K$-factor, defined as the ratio to the LO cross section. 
distributions. Similar comments can be made for the polarized distributions in Fig. 2. Compared with the unpolarized case, they show a milder enhancement of the leading jet distribution and a stronger suppression of the subleading jet distribution.

While single-jet production can be described in terms of the jet pseudorapidity and transverse momentum, the availability of a second jet allows to define more kinematical observables to analyze the underlying partonic kinematics in detail. In that sense, it is instructive to study the unpolarized cross section as a function of the usual dijet kinematical observables $\left\langle p_{T}^{B}\right\rangle_{2}, M_{12}, \eta^{*}$ and $\xi_{2}$, defined in Sec. II, as presented in Fig. 3 .

As it was noted for the kinematics of HERA [33], higher order corrections are sizable for all the variables under consideration. The scale variations of the NLO calculation are as large as the LO ones, or even larger, in the lower bins of the $M_{12},\left\langle p_{T}^{B}\right\rangle_{2}$ and $\xi_{2}$ distributions, as the infrared limit is approached. As mentioned, this behavior is mainly due to the asymmetrical cuts in $p_{T}$ imposed to the two jets. In the Breit frame, LO kinematics implies that the two outgoing partons generating the jets have opposite transverse momentum, and therefore the region with $\left\langle p_{T}^{B}\right\rangle_{2}<$ $5 \mathrm{GeV}$ is not accessible at that order. A similar argument can be used to show that new regions of $M_{12}<10 \mathrm{GeV}$ and low $\xi_{2}$ become accessible only at NLO. This discrepancy in the available phase space at different orders is known to cause instabilities in the perturbative expansion [45]. Actually, for that forbidden phase space region the calculation is effectively a LO one. Note, however, that the use of symmetric cuts in $p_{T}$ leads to even worse perturbative problems, due to the enhancement of large logarithmic contributions related to the back-to-back configuration that can completely spoil the convergence of the expansion $[33,46]$. The NLO corrections show a clear pattern, shifting the distributions to lower values of $\left\langle p_{T}^{B}\right\rangle_{2}<5 \mathrm{GeV}$, which are in turn correlated to lower values of $M_{12}$ and $\xi_{2}$, and higher values of $\eta^{*}$.

In Fig. 4 we show the same distributions of Fig. 3 but for the polarized cross section. In addition to the uncertainty associated to the scale variation, Fig. 4 also presents the uncertainty associated to the polarized PDFs, estimated using the DSSV set of PDFs replicas from [44]. Compared to the unpolarized case, for low $M_{12},\left\langle p_{T}^{B}\right\rangle_{2}, \eta^{*}$ and $\xi_{2}$ it can be seen that while the NLO corrections follow the same pattern, they are generally milder, with lower $K$-factors. There is also a difference in the behavior of the second order corrections for higher values of $M_{12}, \eta^{*}$ and $\xi_{2}$,
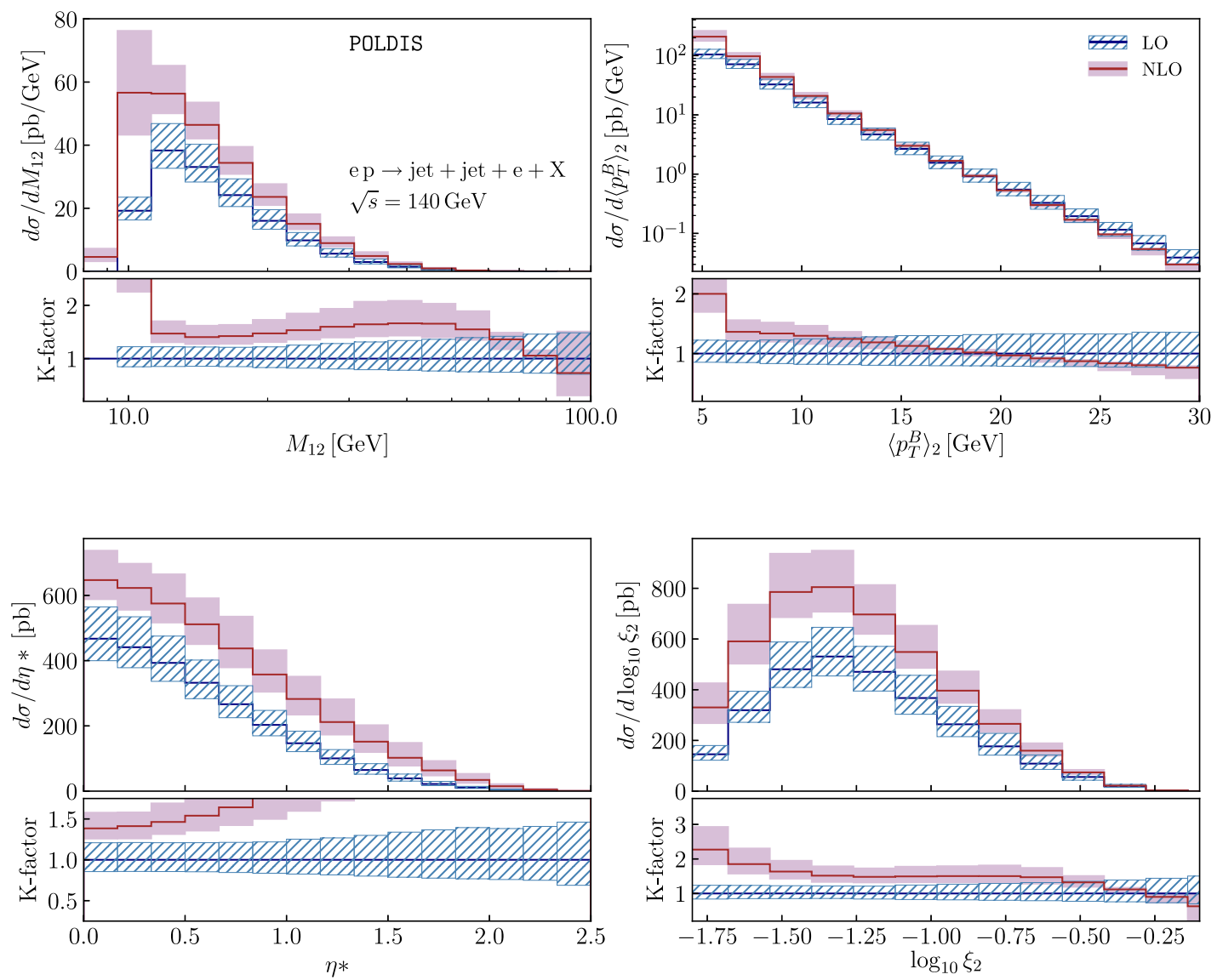

FIG. 3. Inclusive dijet production distributions as a function of the variables $\left\langle p_{T}^{B}\right\rangle_{2}, M_{12}, \eta^{*}$ and $\log _{10}\left(\xi_{2}\right)$. The lower boxes show the $K$-factor for each distribution. 

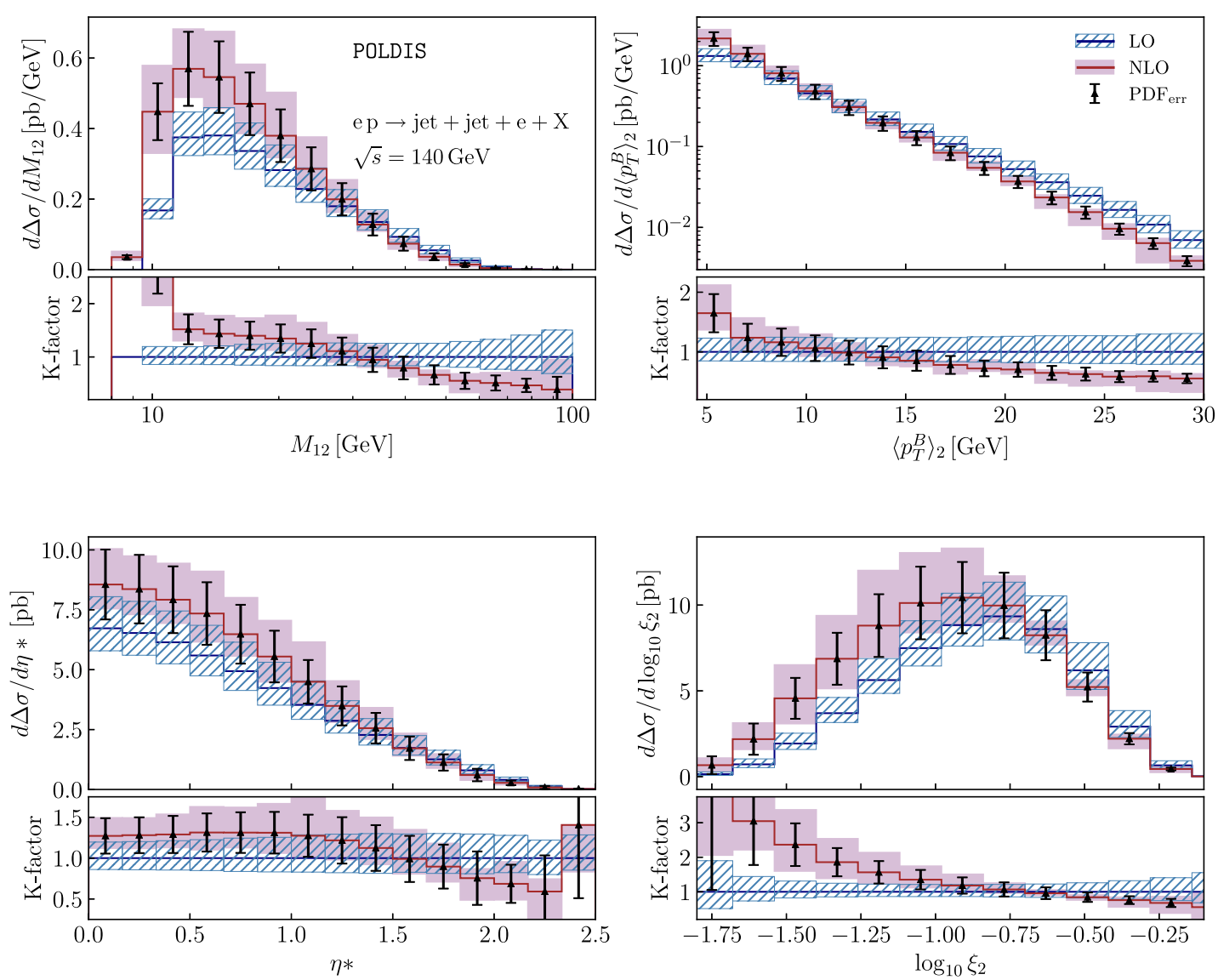

FIG. 4. Same as Fig. 3, but for the polarized case. The uncertainty associated the polarized PDFs is depicted with black error bars, in addition to the theoretical uncertainty from scale variations.

resulting in stronger suppressions than the ones observed in the unpolarized case. The $\xi_{2}$ distribution is particularly shifted toward higher momentum fractions. The same considerations regarding theoretical uncertainties apply to the polarized case, leading to the strong NLO scaledependence. For most of the kinematical range, the PDFs uncertainty is comparable to the scale variation bands, highlighting the importance that higher order corrections will have in more precise extraction of partonic distributions based on EIC observables.

The somewhat big NLO corrections, and the differences between the unpolarized and polarized cases, can be better understood by analysing the previous distribution at different values of $Q^{2}$. As an example, in Figs. 5 and 6 we present the unpolarized and polarized double-differential distribution, i.e., in bins of $Q^{2}$ and $\log _{10}\left(\xi_{2}\right)$, respectively. Regarding the unpolarized distributions of Fig. 5 it can be noted that, as expected, lower $Q^{2}$ values are correlated to smaller momentum fractions, from which the cross section receives its most important contributions. Dijet production measurements at the EIC are therefore expected to explore the mid- $x$ region, $10^{-2}<x<10^{-1}$. The NLO cross sections for the high $Q^{2}$ bins are in good agreement with the LO calculations and show small scale dependence, indicating good convergence of the perturbative series. In addition to the complementary constraints on the quarks polarized and unpolarized distribution functions, restrictions coming from this region on the gluon helicity distribution, which is mainly probed down to $x \sim 5 \times 10^{-2}$ by RHIC data, will be specially important. On the other hand, in Fig. 5 it can be seen that both the $K$-factors and theoretical uncertainties increase as lower $Q^{2}$ values are considered. This is consistent with the aforementioned population of the new phase space region at low $\xi_{2}$ becoming available at NLO.

Compared to the unpolarized case, the polarized distributions of Fig. 6 present two striking features: they decrease at lower $Q^{2}$, and they also display significant differences in shape between LO and NLO results in that region. Both features can be explained by the analysis of the contributions from the quark and gluon channels to the polarized cross section. In Fig. 7 we present, precisely, the dijet double-differential polarized and unpolarized distribution as a function of $Q^{2}$ and $\log _{10}\left(\xi_{2}\right)$, distinguishing the contributions initiated by the quark and gluon channels. In this case, the lower insets in the plot depict the ratio between the gluon- and quark-initiated differential cross sections. Compared to the unpolarized case, for which both 

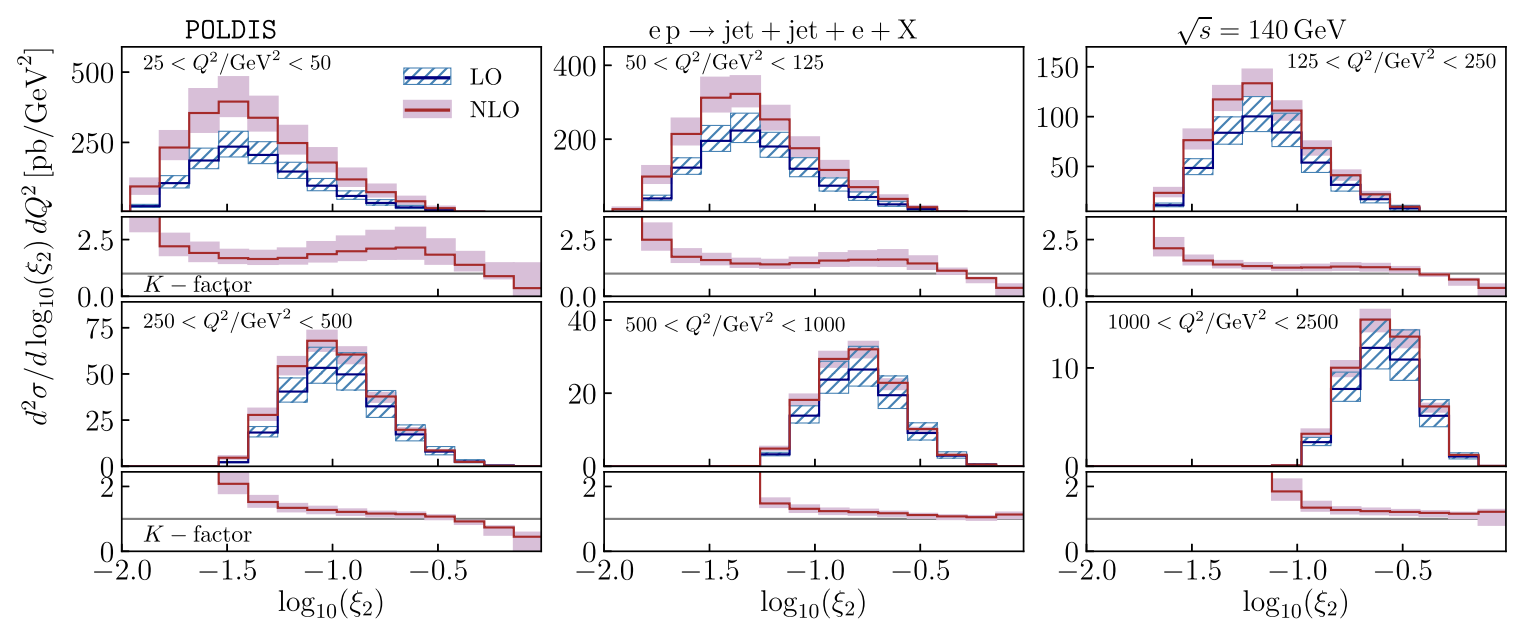

FIG. 5. Inclusive dijet production unpolarized cross sections in bins of $Q^{2}$, as a function of $\log _{10}\left(\xi_{2}\right)$. The LO and NLO uncertainty bands are obtained as in Fig. 4. The lower panels display the $K$-factors to the LO calculation.
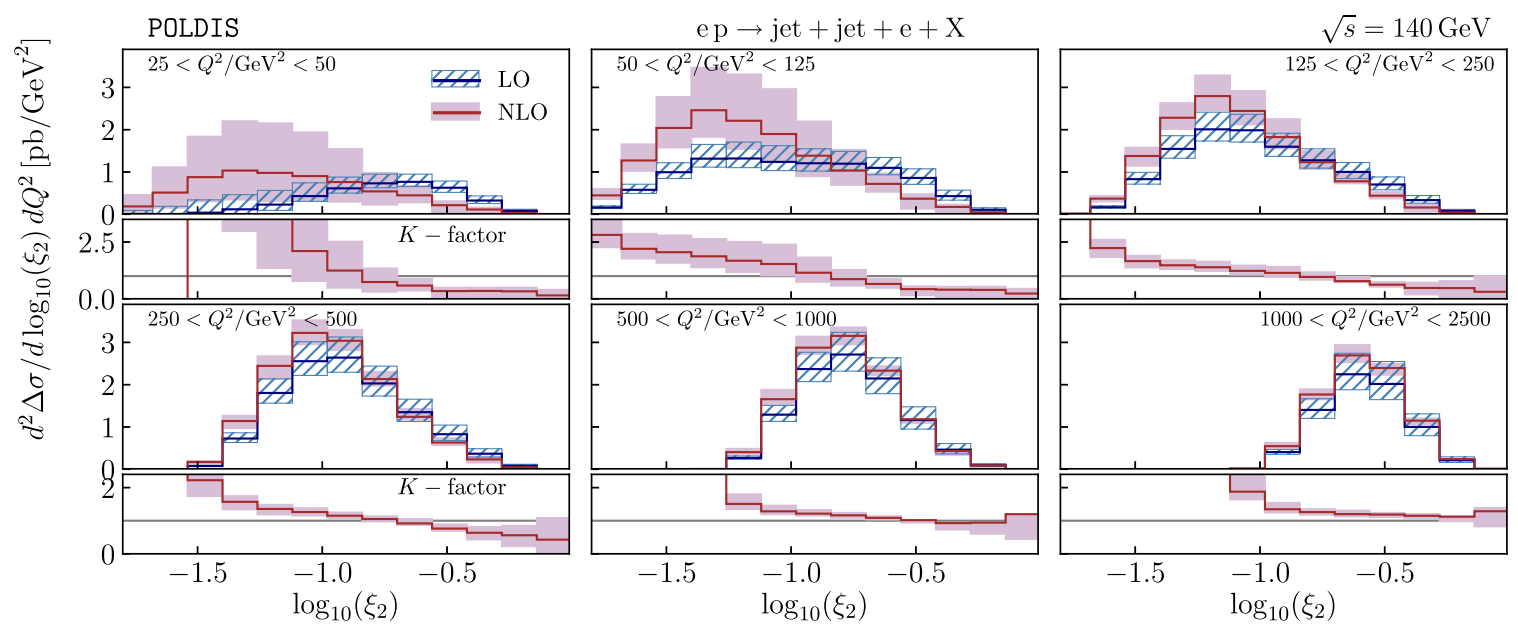

FIG. 6. Same as Fig. 5, but for the polarized case.

the gluon and quark-initiated contributions are positive, the peculiar behavior of the polarized cross sections as a function of $Q^{2}$ can be traced back to the negative sign of the gluon contribution below $Q^{2}=600 \mathrm{GeV}$, which becomes more significant for lower values of $Q^{2}$, as shown in the ratio between the gluon and quark contributions. The enhancement of the negative contribution from the gluonic channel at low- $Q^{2}$ leads to strong cancellations against the positive quark contributions, and therefore to a reduction in the polarized cross section. At the same time, this reduction leads to larger relative theoretical uncertainties. The cancellation between channels is also responsible for the change in the behavior observed in the $\xi_{2}$ distributions in Figs. 4 and 6 with respect to the unpolarized case, the different shapes of the NLO corrections in the distributions of the other kinematical variables and the somewhat large uncertainty arising from the polarized partonic distributions. Fig. 7 also shows that the NLO shift in the polarized $\xi_{2}$ distribution of Fig. 4 is mostly associated to a shift in the quark contribution toward lower momentum fractions.

The most relevant observables in polarized processes are the double spin asymmetries, defined as the ratio of the polarized and unpolarized cross sections $A_{L L}=\Delta \sigma / \sigma$, since the cancellation of systematic uncertainties is expected to happen in the quotient. In Fig. 8 we show the double spin asymmetries and the associated theoretical uncertainties for $\left\langle p_{T}^{B}\right\rangle_{2}, M_{12}, \eta^{*}$ and $\xi_{2}$, obtained performing simultaneous scale variations in both the numerator and the denominator. The asymmetries values are typically of order $\sim(1-10 \%)$ in the relevant regions, with a significant reduction in the NLO values due to the higher $K$-factors observed in the unpolarized cross sections. The only exception is the $\xi_{2}$ distribution, where the unusual behavior due to gluon cancellation and the shift in the quark contribution to the polarized cross section leads to an enhancement in the asymmetry at lower momentum 

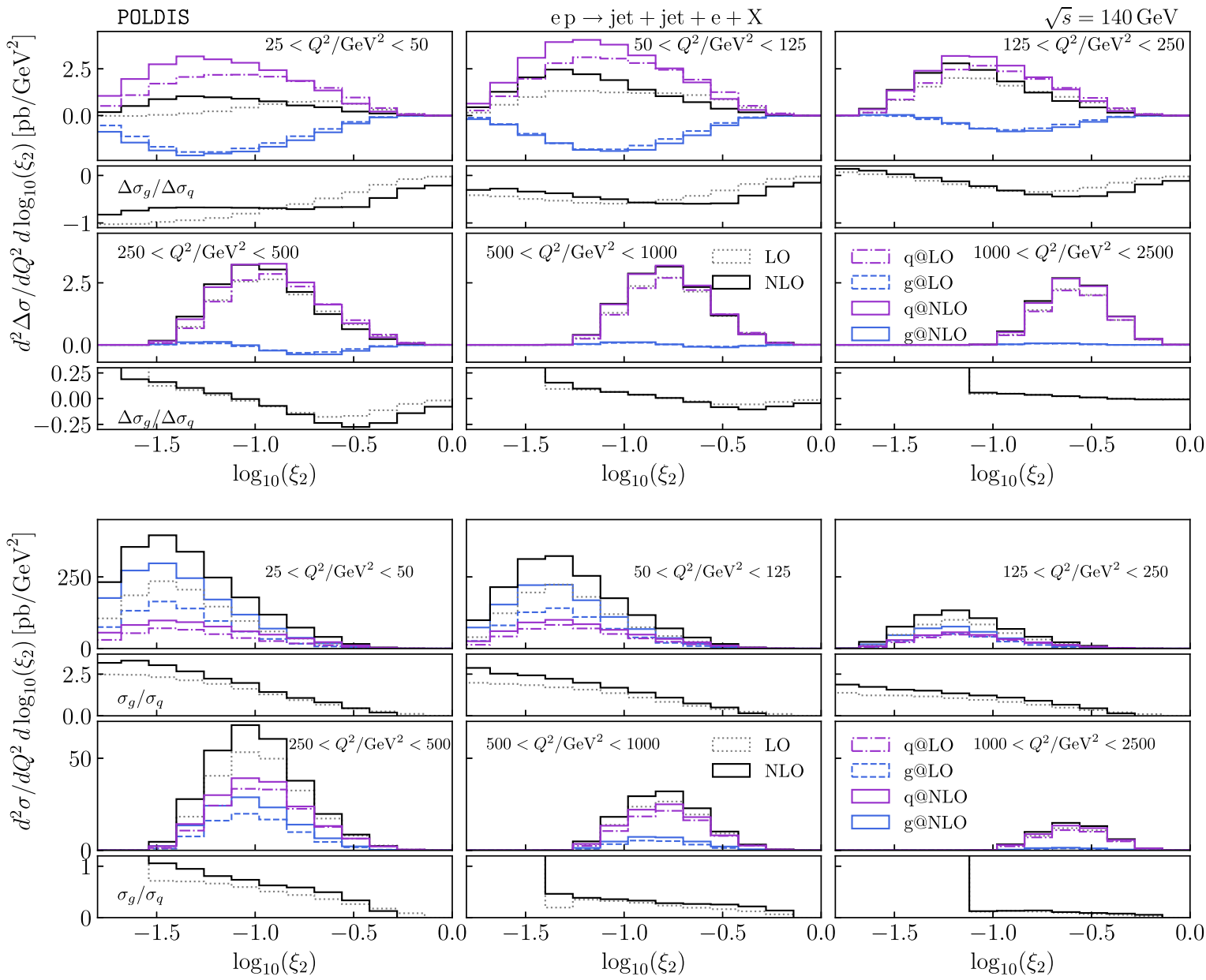

FIG. 7. Same as Figs. 5 and 6, but separating the contributions initiated by quarks and gluons to the dijet cross section, both at LO (dashed lines) and at NLO (solid lines). The lower insets show the ratio between the gluonic and quark contributions to the cross section.

fractions, albeit the very small values of the asymmetry in that region.

Once again, the behavior of the asymmetries can be better understood by studying the double-differential $Q^{2}$ dependence of the distributions. Figure 9 depicts the double spin asymmetry as a function of both $Q^{2}$ and $\xi_{2}$. The reduction of the polarized cross sections for low values of $Q^{2}$ due to the negative gluonic contribution leads to a sizable suppression of the asymmetry in those bins for $\xi_{2} \gtrsim 10^{-1}$. As expected, a stronger dependence on the renormalization and factorization scales is observed in the low $Q^{2}$ region, while a better convergence of the perturbative series is obtained as higher values of $Q^{2}$ are approached. It is worth mentioning that, for the first two bins of $Q^{2}$, the significant shift in the NLO quark contribution toward lower momentum fractions shown in Fig. 7 results in an enhancement of the asymmetries for $\xi_{2} \lesssim 10^{-1}$. The clear pattern of the NLO corrections to the asymmetries can be easily understood by the direct comparison of the Figs. 5 and 6: high $Q^{2}$ bins show $K$-factors close to 1 , due to the smaller corrections observed for both the polarized and unpolarized distributions. The difference in sign of the NLO corrections for each case in the high momentum fraction region results in the reduction of the asymmetries shown in Fig. 9, which becomes more important as lower values of $Q^{2}$ are reached. This very same behavior has been seen for the other kinematical observables $M_{12},\left\langle p_{T}^{B}\right\rangle_{2}$ and $\eta^{*}$.

\section{POLARIZED NNLO INCLUSIVE-JET PRODUCTION}

Having discussed our NLO dijet production calculation, we can now turn to the NNLO corrections for single jet production, obtained through the application of the P2B method. In this section, we present our results for polarized single-inclusive jet production at NNLO in the laboratory frame (L), for the Electron-Ion-Collider kinematics. Similarly to [18], the default distributions are obtained reconstructing the jets with the anti- $k_{T}$ algorithm and $E_{T}$-scheme recombination, using a jet radius $R=0.8$, and fixing the normalization and factorization scales central values as $\mu_{F}^{2}=\mu_{R}^{2}=Q^{2} \equiv \mu_{0}^{2}$. As in the previous section, $\alpha_{s}$ is evaluated at NLO accuracy with $\alpha_{s}\left(M_{z}\right)=0.118$. 

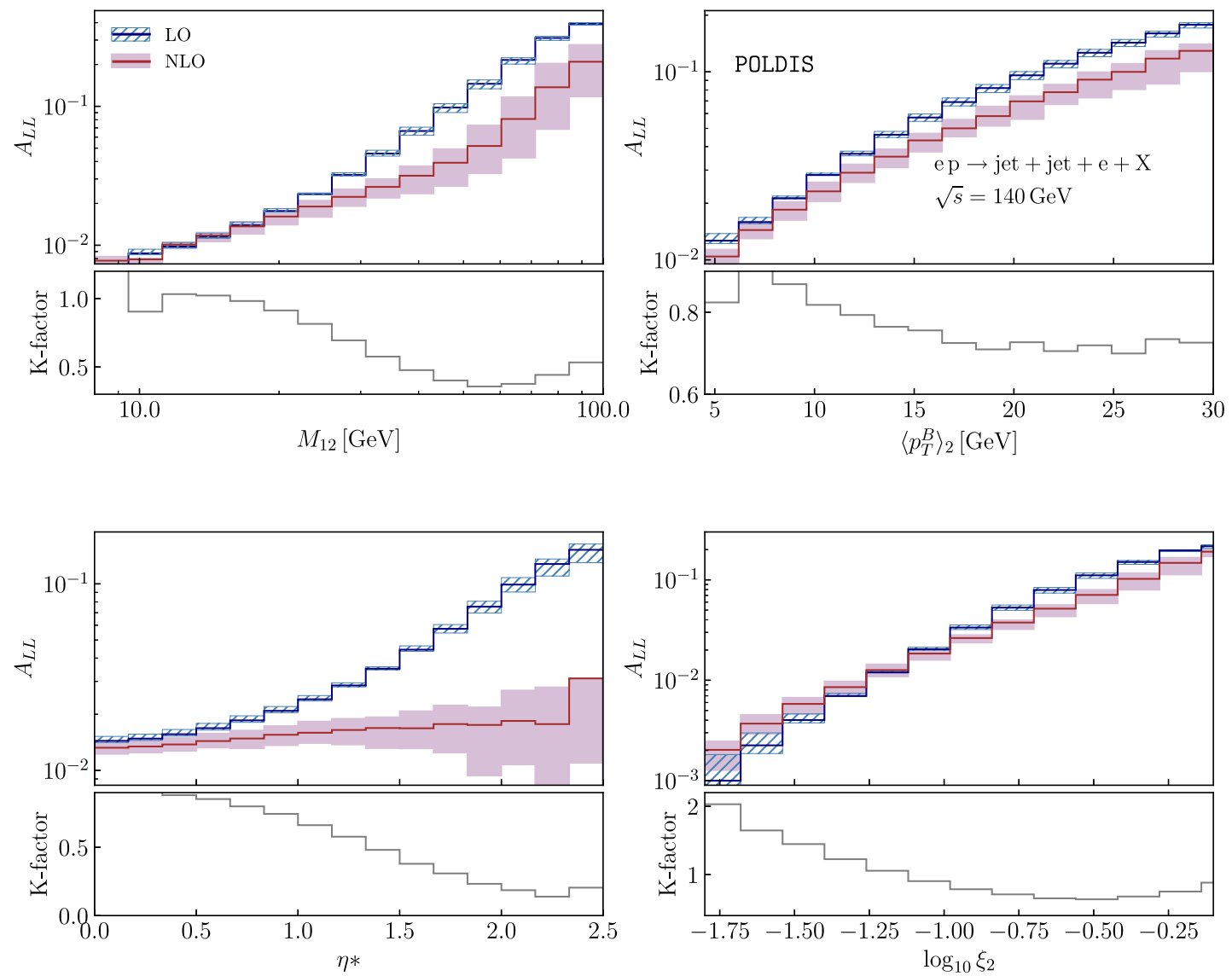

FIG. 8. Double spin asymmetries for dijet production, as a function of $\left\langle p_{T}^{B}\right\rangle_{2}, M_{12}, \eta^{*}$ and $\xi_{2}$. The lower boxes depict the $K$-factors to the LO calculation.
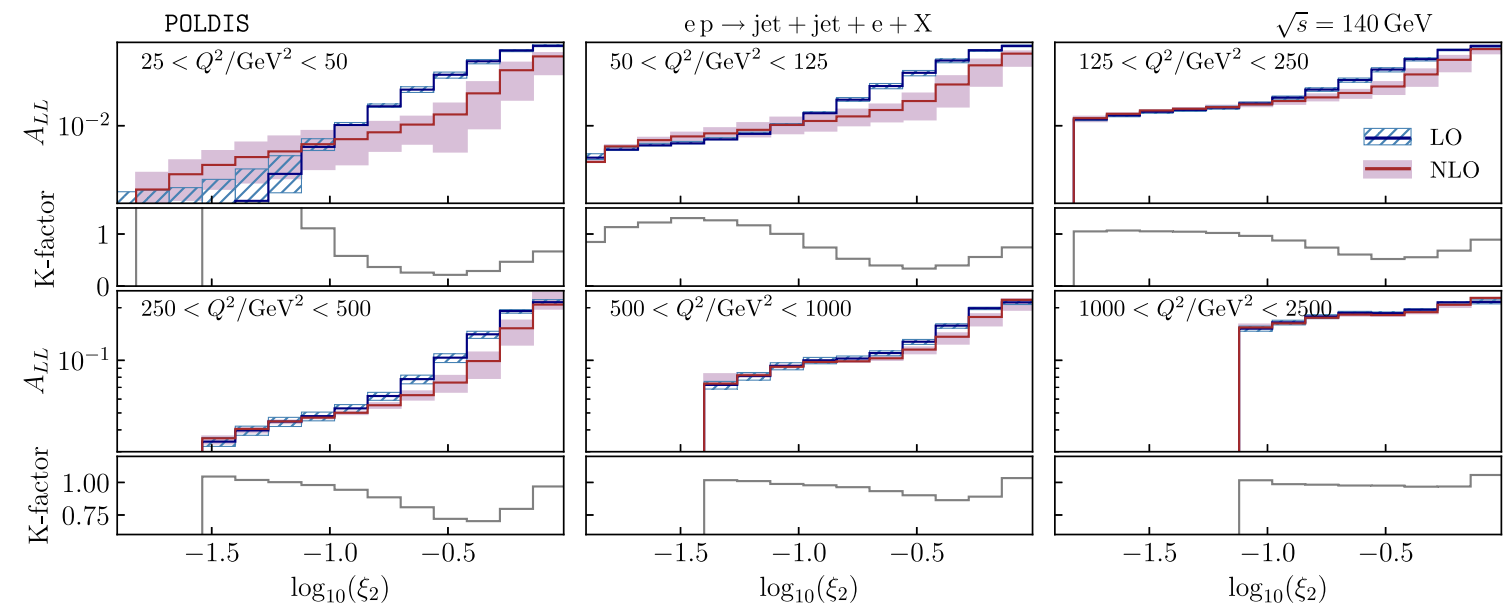

FIG. 9. Double spin asymmetry as a function of $\log _{10}\left(\xi_{2}\right)$ and $Q^{2}$. As in the previous cases, the inset lower boxes show the $K$-factor to the LO asymmetry.

The reconstructed jet in the laboratory frame is then required to satisfy:

$$
\begin{aligned}
5 \mathrm{GeV} & <p_{T}^{L}<36 \mathrm{GeV} \\
\left|\eta^{L}\right| & <3
\end{aligned}
$$

while on the leptonic side we impose the additional cuts:

$$
\begin{aligned}
0.04 & <y<0.95, \\
25 \mathrm{GeV}^{2} & <Q^{2}<1000 \mathrm{GeV}^{2} .
\end{aligned}
$$


The lower cut in $Q^{2}$ was chosen to avoid differences in the phase space available at different orders. Note that at LO the transverse momentum of the jet in the laboratory frame is given by $\left(p_{T}^{L}\right)^{2}=Q^{2}(1-y)$, and thus the region $Q^{2} \lesssim$ $25 \mathrm{GeV}^{2}$ is kinematically forbidden for the specified cuts in $p_{T}^{L}$. Since there is no NNLO global fit of polarized PDFs available, the parton distributions sets used were, once again, the NLO extractions NLOPDF4LHC15 [43] and DSSV $[3,44]$ for the unpolarized and polarized case, respectively.

In Fig. 10 we present the cross section for singleinclusive jet production in polarized DIS, as a function of the jet transverse momentum $p_{T}^{L}$, its pseudorapidity $\eta^{L}$, and in terms of $Q^{2}$ and $x$, calculated at LO, NLO and NNLO accuracy. The lower insets in Fig. 10 show the $K$-factors, defined as the ratios to the previous order, that is, $K^{\mathrm{NNLO}}=\sigma^{\mathrm{NNLO}} / \sigma^{\mathrm{NLO}}$ and $K^{\mathrm{NLO}}=\sigma^{\mathrm{NLO}} / \sigma^{\mathrm{LO}}$. As in the case of dijet production, the theoretical uncertainty bands were obtained performing a seven-point independent variation of the renormalization and factorization scales as $\mu_{R}, \mu_{F}=\left[\frac{1}{2}, 2\right] \mu_{0}$. The uncertainty associated to the polarized parton distributions was estimated using the DSSV set of PDFs replicas. Note that due to the unavailability of proper polarized NNLO PDF, this bands should be taken only as a first attempt to quantify the nonperturbative errors in the NNLO cross section. The same NLO PDFs were used at all orders so as to quantify only the variations arising from the perturbative calculation.

As it can be seen in Fig. 10, the main effect of higher order corrections is to shift these distributions toward higher values of pseudorapidity and lower values of transverse momentum, since more jets originating from the emission of additional partons become available in those regions. In the case of the pseudorapidity distribution, this is translated into high values of the NLO $K$-factor in the forward region $\left(\eta^{L}>1\right)$, while a strong suppression in the backward region $\left(\eta^{L}<-1\right)$ is observed. NNLO corrections have the same behavior, albeit with lower
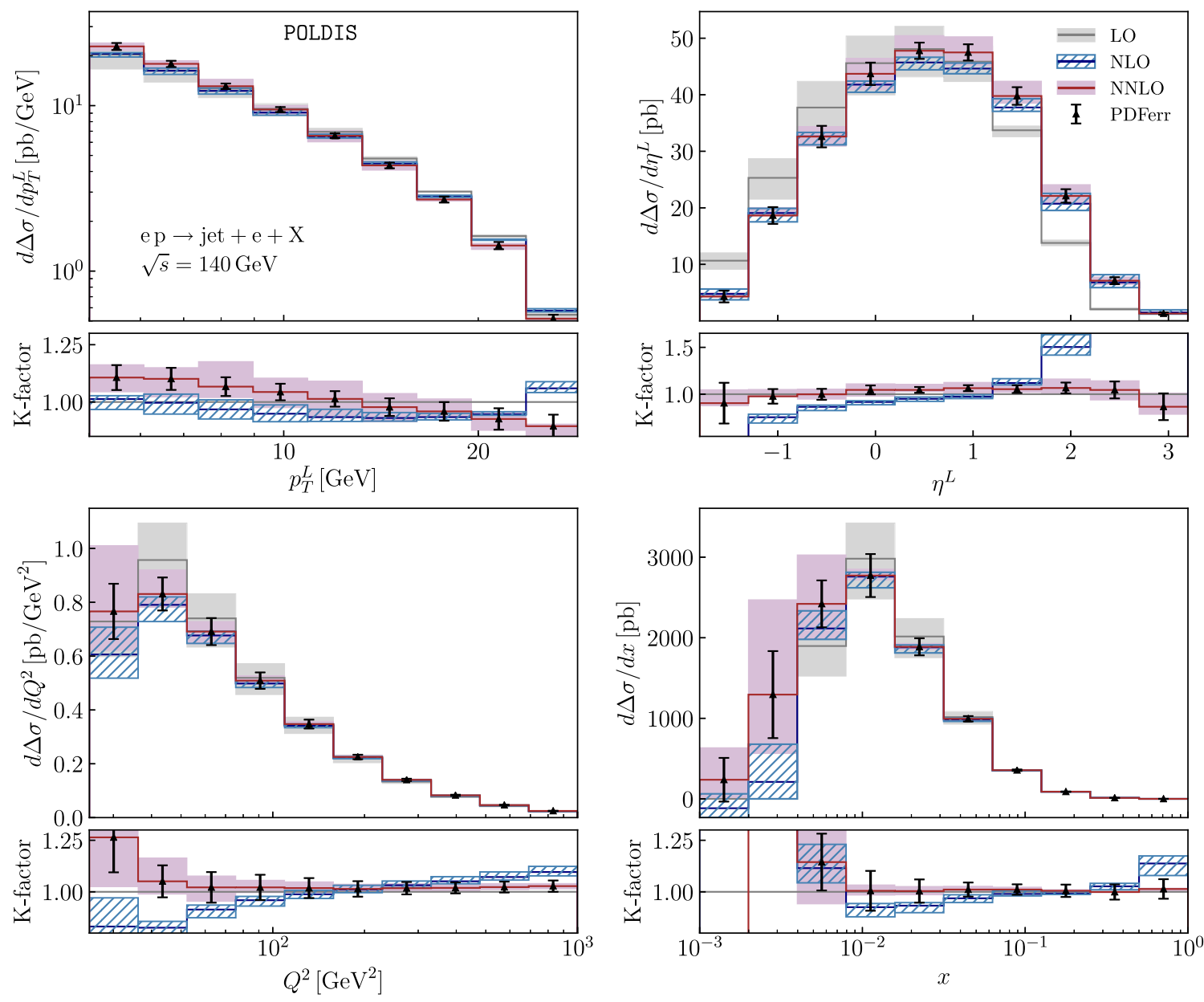

FIG. 10. Polarized single-jet cross section, as distributions in transverse momentum $p_{T}^{L}$, pseudorapidity $\eta$, photon virtuality $Q^{2}$ and Bjorken variable $x$ at LO, NLO and NNLO. The bands reflect the seven point variation in the cross section when independently changing the scales as $\mu_{R}, \mu_{F}=[1 / 2,2] \mu_{0}$. The error bars correspond to the PDF's errors in the NNLO result. The lower inset shows the corresponding $K$-factors, as defined in the main text. 
values of $K$-factor. Similar comments can be made regarding the transverse momentum distribution, which is enhanced for lower values of $p_{T}^{L}$.

For the $p_{T}$ distribution, the NNLO corrections are typically of order $10 \%$, while for the $\eta$ distributions they are of order $5 \%$. It should be noted that while there is good agreement between the NLO and NNLO calculations, with overlapping bands throughout the kinematical range, anticipating convergence of the perturbative series, the scale bands for the NNLO distributions are still somewhat large in certain bins compared with those of the NLO. This effect is associated with the kinematical suppression of the LO contributions in some regions due to the cut enforced in $y$ and $p_{T}^{L}$, which spoils the accuracy of the perturbative series in that region. This can be better observed in the $Q^{2}$ and $x$ distributions in Fig. 10. At low values of $Q^{2}$ and $x$ the suppression of the Born cross section clearly correlates with higher $K$-factors and scale bands, especially for $x \lesssim 5 \times 10^{-3}$, where the LO is completely forbidden. Other choices of the renormalization and factorization scales central values, e.g., $\mu_{0}^{2}=\left(Q^{2}+\left(p_{T}^{L}\right)^{2}\right) / 2$, lead to a similar behavior of the scale bands. The same effect is also present in the unpolarized case for the aforementioned regions.

Even though the growth of the uncertainty bands at $\mathrm{NNLO}$ in the $p_{T}^{L}$ and $\eta$ distributions originates from the difference in the available phase space at each order, the sizes of the bands in this region are further enhanced in the polarized case compared to the unpolarized one. This results in bigger NNLO bands in $p_{T}^{L}$ and $\eta^{L}$ distributions, as observed in [18]. This enhancement is related to the fact that in the polarized case there are cancellations between processes initiated by different partons. To highlight this point, in Figs. 11 and 12 we present the contributions of the most relevant parton channels to the polarized and unpolarized cross section, respectively. In the polarized case, for most of the explored $Q^{2}$ and $x$ values, the cross section is dominated by initial $u$ quark contribution. However, as lower values of both $Q^{2}$ and $x$ are reached, there are significant cancellations between the $u$ quark channel and the negative contribution of the $d$ quark and gluon channels, which accounts for higher relative uncertainties once the sum over of all the initial parton contributions is taken (the $s$ quark also has a negative contribution, but it is
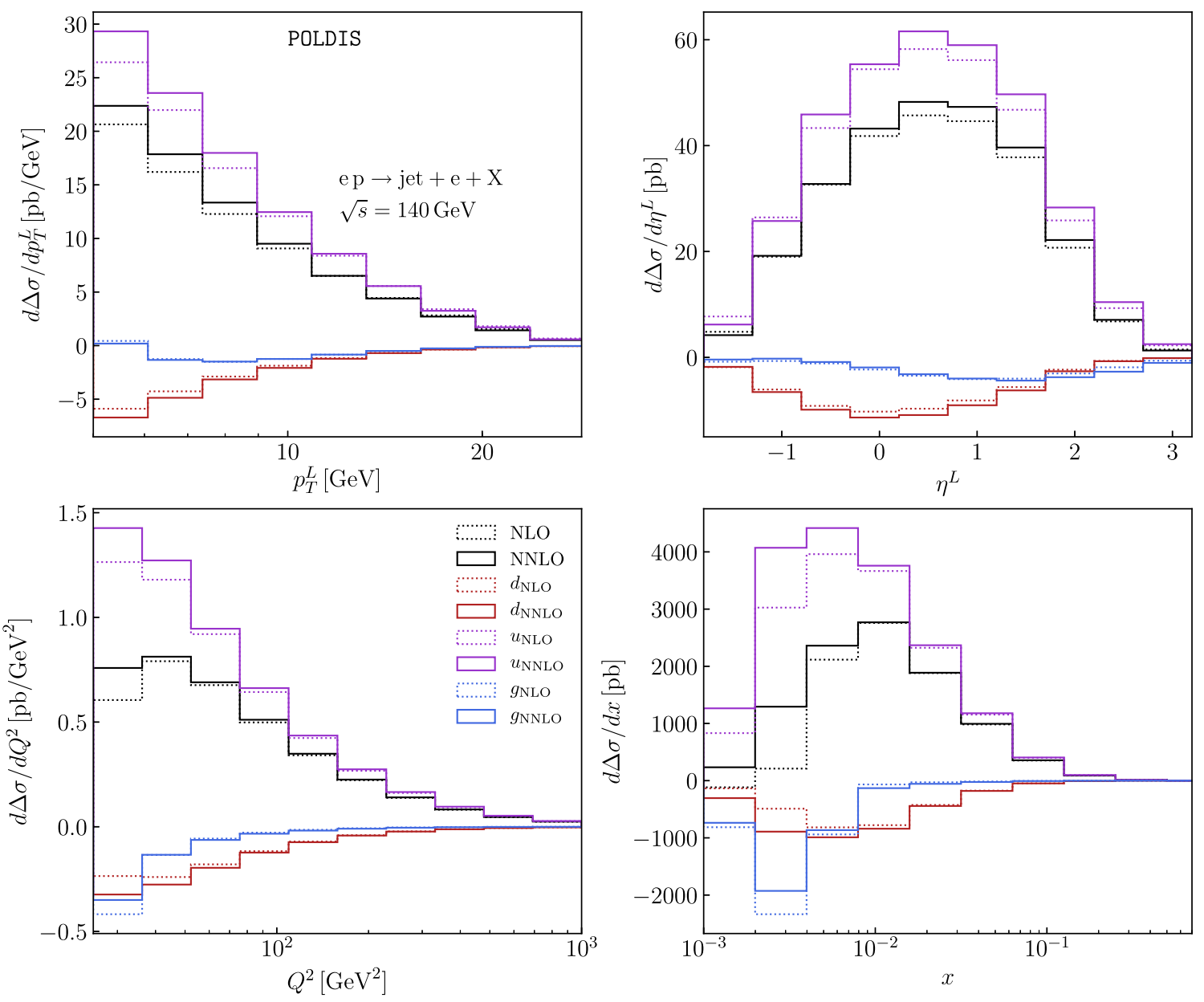

FIG. 11. Same as Fig. 10, but discriminating the contributions to the cross section coming from initial quarks and gluons. 

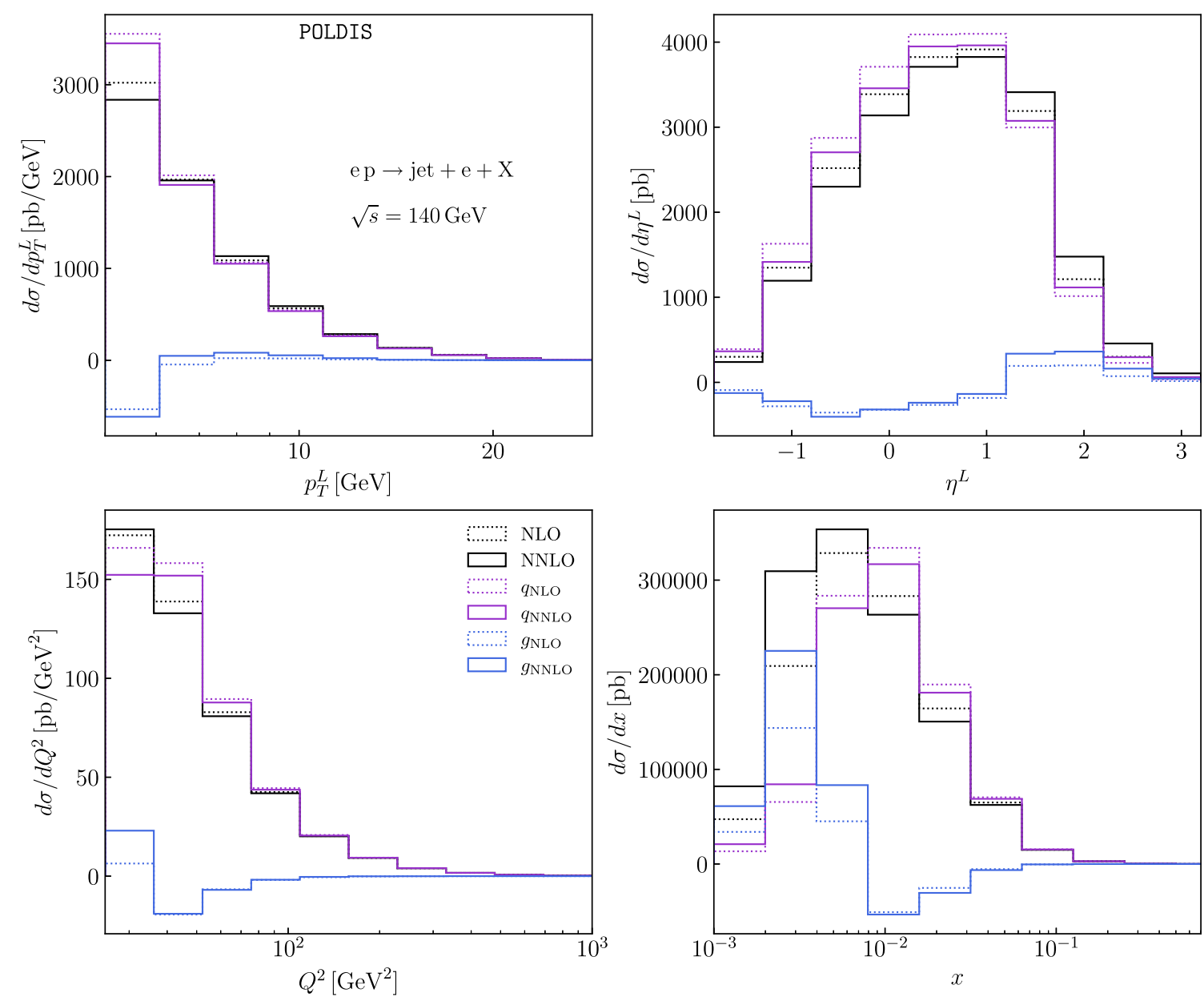

FIG. 12. Same as Fig. 11, but for the unpolarized case.

negligible). Since low $Q^{2}$ and $x$ correlate with low $p_{T}^{L}$ and $\eta^{L} \gtrsim 0$, those same cancellations are translated into the sizable NNLO scale bands in Fig. 10 in those ranges. The unpolarized distributions in Fig. 12, by contrast, do not show such cancellations, since all the quark flavors contribute positively to the cross section and, most importantly, even though the gluon contribution is negative for some portions of the phase space due to collinear factorization counterterms, it is positive at low $x$, where the contribution is most relevant.

For the polarized case, it is worth noticing that, even though it is expected to have a greater gluon contribution at low $p_{T}^{L}$, since that region correlates with low $Q^{2}$, the first bin of the $p_{T}^{L}$ distribution is very small and slightly positive (as opposed to the $u$ and $d$ quarks contributions). This is related to the fact that the gluon contribution to the structure function is positive below $x \sim 2 \times 10^{-2}$. Since the structure function is obtained by the integration over all the $p_{T}^{L}$ range, as lower values of $p_{T}^{L}$ are reached the $p_{T}^{L}$ distribution must become positive at some point.

Regarding the uncertainty associated to the PDFs in Fig. 10, it is typically of order $5 \%-10 \%$ for the region of
$\left\{p_{T}^{L}, \eta^{L}\right\}$ studied. Though this uncertainty is comparable to the NNLO corrections for most of the kinematical range, it should be noted that for the low $p_{T}^{L}$ region, it becomes smaller than the NNLO corrections, highlighting the relevance that NNLO extractions will have in order to match the accuracy of the perturbative side. As in the case of the scale-variations bands, the PDF uncertainty becomes larger as lower values of $x$ and $Q^{2}$ are approached, since the cancellation between the different partonic channels for those bins is sensitive to changes in the partonic distributions.

In the upper panels of Fig. 13 we present the double spin asymmetries as a function of $p_{T}^{L}$ and $\eta^{L}$. Once again, the scale bands are obtained by the simultaneous seven-point variation of the scales in both cross sections. Its most striking feature is the important NNLO suppression of the asymmetry in the high pseudorapidity region, with milder corrections for intermediate $\eta^{L}$. The different behavior of the NNLO corrections to the asymmetry can be traced back to the change of sign of the gluon-initiated contributions for the polarized and unpolarized cross sections, which start at $\mathcal{O}\left(\alpha_{S}\right)$. To highlight this point, in the lower panels of 

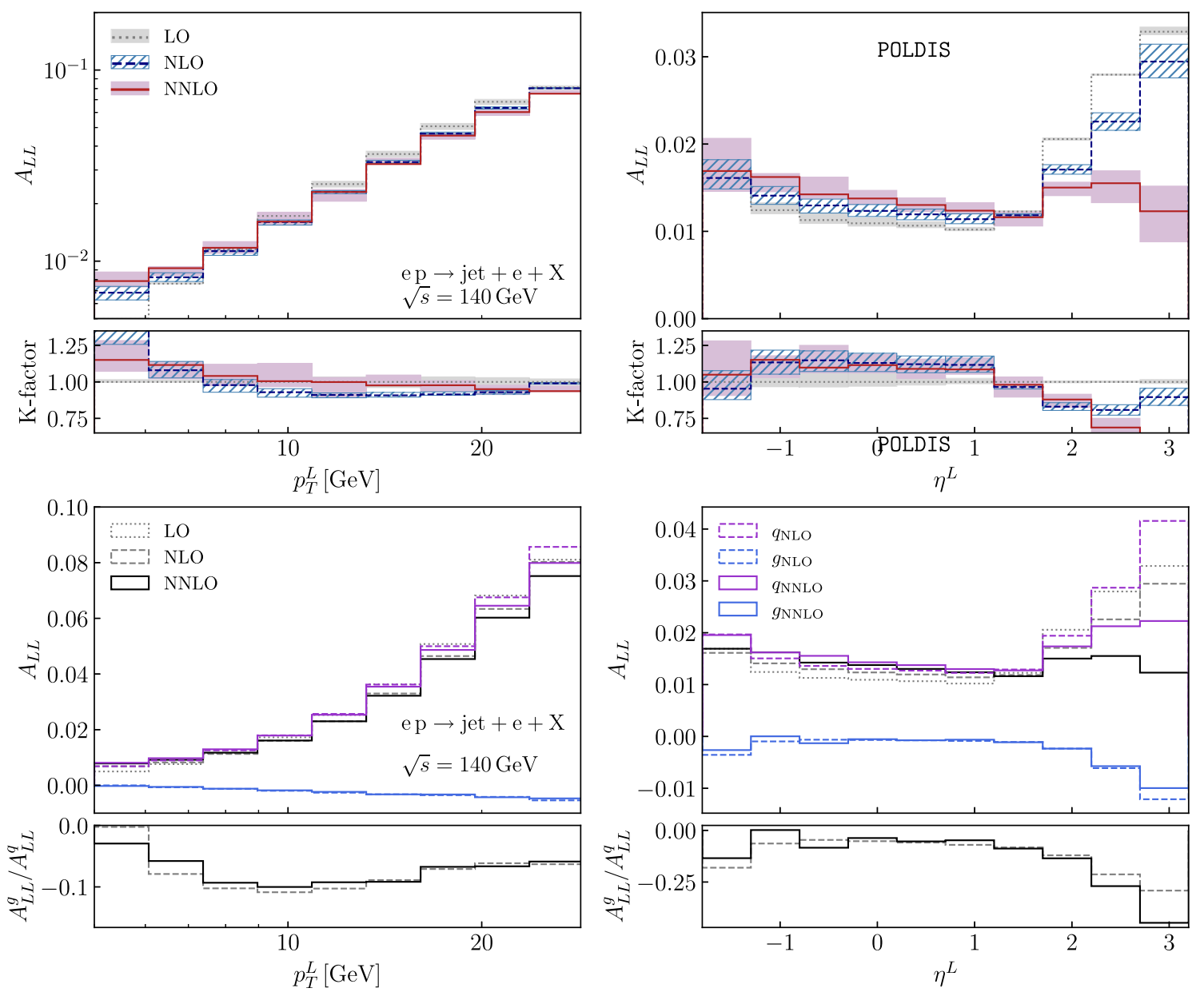

FIG. 13. Double spin asymmetries and theoretical uncertainties up to NNLO (upper panels) and separation into partonic channels (lower panels) as distributions of $p_{T}^{L}$ and $\eta$. For the upper panels the insets shows the corresponding $K$-factors, as defined in the main text, while for the lower ones, the insets present the ratio between the gluonic and quark contributions to the asymmetry.

Fig. 13 we also present the quark and gluon-initiated contributions to the double spin asymmetry in $p_{T}^{L}$ and $\eta^{L}$. The decomposition in partonic channels is made at the numerator level, i.e., setting to zero the gluon or quark polarization, but keeping all the contributions in the denominator. While there are cancellations already present at NLO at the higher pseudorapidities, the NNLO enhancement of the gluon-initiated contribution to the cross section (which is, effectively, a NLO one) for $\eta^{L} \gtrsim 1$, is translated in a reduction of the quark contributions to the asymmetry for that region and results in the foregoing suppression of the double spin asymmetry. Note that higher values of pseudorapidity are correlated to low $x$, where the gluon contribution is most relevant and resummation of large logarithmic corrections would be needed. It is also worth mentioning that, although milder, sizable corrections are obtained for $\eta^{L} \lesssim 1$ and the low $p_{T}^{L}$ region.

Another feature associated to cancellation between partonic channels in the polarized cross section is the reduced dependence on the parameters of the jetreconstruction algorithm, compared to the unpolarized case. To emphasize this point, in Fig. 14, we present the NNLO cross sections as a distribution of both $p_{T}^{L}$ and $\eta^{L}$, for different values of the jet radius $R=0.5,0.8,1$ used in the anti- $k_{T}$ algorithm. In both cases, higher values of jet radius correspond to larger cross sections in the whole kinematical range due to the inclusion of more jets that satisfy the imposed cuts. However, the polarized case shows a reduced dependence in $R$ at low $p_{T}^{L}$ and the intermediate $\eta^{L}$ values, precisely where the stronger cancellations between channels take place. This results in an overall reduction of the dependence of the polarized cross section on the jet parameter. It is worth noticing that while the total cross section is affected by these strong cancellations between channels, with the use of jet tagging techniques $[15,16]$ it could be possible to noticeably modify the shape of the distributions, enhancing the contributions from different partons.

The difference of sensitivity to changes in the jet radius will in turn modify the behavior of the double spin asymmetries. In Fig. 15 we present the NNLO double spin asymmetries in the $p_{T}^{L}$ and $\eta^{L}$ distributions for the $R$ 

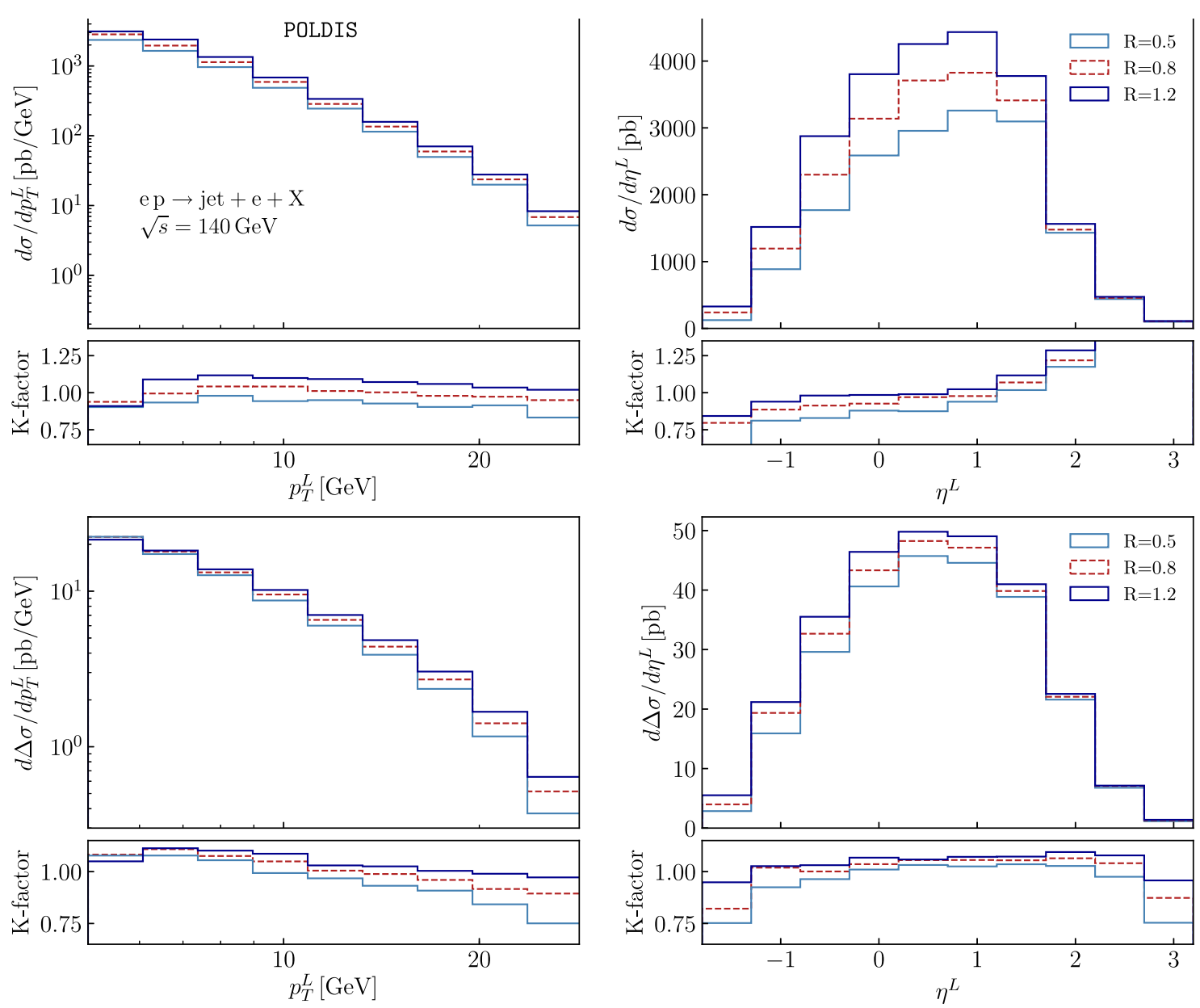

FIG. 14. NNLO polarized and unpolarized cross sections as a distribution of $p_{T}^{L}$ and $\eta^{L}$ for different choices of the jet radius $R$ value.
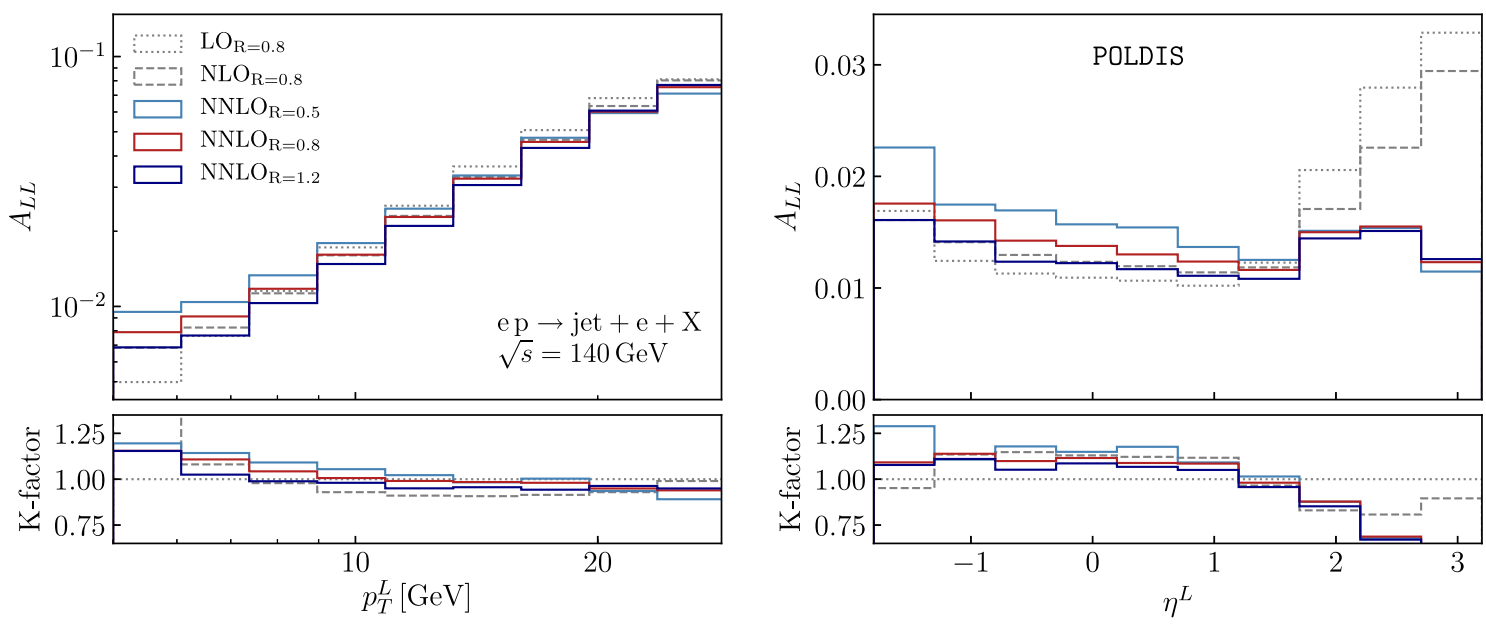

FIG. 15. NNLO double spin asymmetries as a distribution of $p_{T}^{L}$ and $\eta$ for different choices of the jet radius $R$ value.

values used before. As expected, a larger dependence on $R$ is obtained in those regions where the cancellation between channels for the polarized cross sections are more important. For those regions, the increase in $R$ leads to a relative increase of the unpolarized cross section, and consequently to a reduction in the spin asymmetry. Conversely, lower values of $R$ produce an increment of the asymmetry in the same regions. Figure 15 also shows the LO and NLO asymmetries for $R=0.8$. Note that for $\eta^{L} \lesssim 1$ and $p_{T} \lesssim 10$, the variations with the jet radius are greater than 
those coming from the perturbative series. The jet parameters are therefore expected to have sizable impact in the double spin asymmetries in regions where cancellation between partonic contributions take place in the polarized cross section.

\section{CONCLUSIONS}

In this paper we have presented the NLO calculation for the production of dijets in polarized and unpolarized lepton-nucleon DIS in the Breit frame, for the EIC kinematics. Our calculation is based in a generalization of the dipole subtraction method to handle the polarization of initial-state particles, which is discussed in detail. The cross sections were studied as functions of the leading jets transverse momenta $p_{T, 1}^{B}$ and $p_{T, 2}^{B}$, invariant mass of the jets $M_{12}$, the mean transverse momentum $\left\langle p_{T}^{B}\right\rangle_{2}$, the difference in pseudorapidities $\eta^{*}$ and the dijet momentum fraction $\xi_{2}$. Additionally, the double-differential distributions in $Q^{2}$ and $\xi_{2}$ were analyzed. Both for the polarized and unpolarized cross sections, the differential distributions show important NLO corrections, particularly for low values of $M_{12}$ and $\xi_{2}$, and higher values of $\eta^{*}$, associated to differences in the phase space available at each order. While the NLO corrections obtained show good agreement with the LO calculations and reduced dependence on the choice for the factorization and renormalization scales, for values of $Q^{2}$ above $250 \mathrm{GeV}$, anticipating convergence of the perturbative expansion, the distributions for lower values of $Q^{2}$ present sizable corrections as well as a strong dependence on the scale choice. We noted that this effect is further enhanced in the polarized cross sections, due to the nonnegligible negative contribution of the gluon-initiated channel, producing noticeable differences between the polarized results and their unpolarized counterparts. This difference in behavior is translated to the double spin asymmetries, with significant suppression in $M_{12}, \eta^{*}$ and $\left\langle p_{T}^{B}\right\rangle_{2}$. Once again, the corrections are more significant as lower values of $Q^{2}$ are approached.

The dijet calculation was in turn used to obtain the polarized NNLO single-inclusive jet production crosssection in the laboratory frame via the P2B method [18], which combines the exclusive NLO dijet cross section along with the inclusive NNLO polarized structure function. We expanded on our previous results to include a better estimate of the theoretical uncertainty, as well as the dependence on the jet radius. Good agreement was found between the NLO and NNLO results for the range studied in $p_{T}^{L}$ and $\eta^{L}$. The somewhat large size of some of the NNLO uncertainty bands was linked to a combination of the effects due to the difference in phase space available at LO at low $Q^{2}$ and $x$, also present in the unpolarized case, as well as the cancellation between partonic channels in the polarized cross section. This channel cancellation also leads to a reduced dependence of the polarized cross section in the jet radius $R$, which in turn produces a more noticeable dependence of the double spin asymmetries in $R$ in the regions of low $p_{T}^{L}$ and intermediate values of $\eta^{L}$. This hints toward a sizable dependence of the polarized cross section and asymmetries with the jet parameters in those regions, as well as important sensibility to the recently proposed jet-tagging techniques.

The results presented on this paper highlight the relevance that higher order QCD corrections will have in the precise description of the jet observables to be obtained in the future EIC, as well as the potential of those measurements to further improve our understanding of the spin structure of the proton and, particularly, in the precise extraction of polarized parton distributions.

\section{ACKNOWLEDGMENTS}

We thank Rodolfo Sassot for discussions and Stefano Catani, Gavin Salam and Mike Seymour for useful communications. This work was partially supported by CONICET and ANPCyT.

\section{APPENDIX A: DIPOLE BUG IN DISENT}

The presence of a bug in the gluon channel in DISENT was reported long ago in [35-38], particularly while studying the event shape distributions in DIS. After a careful analysis, along with an extensive comparison with DISASTER [47] (a code which showed good agreement with resummed event shape calculations), and also by writing independent codes, we found that the Born matrix element used in one of the dipole subtraction terms in the gluon channel had the momentum of two final-state partons interchanged, leading to the reported discrepancies. Due to the nature of the bug, it turns out to produce noticeable differences only in certain extreme regions of the phase space, and remains within the typical statistical uncertainties of the calculations in many others.

We have checked that the fixed counterterm actually corrects the reported disagreement between DISENT and DISASTER in the event shapes, as well as the differences between DISENT and the analytical calculation for logarithmically enhanced terms. As an example, we present in Fig. 16 the difference between the $\mathcal{O}\left(\alpha_{S}^{2}\right)$ coefficient for the fixed-order Monte Carlo calculation and the expansion of the resummed calculation for the gluonic contribution to $\tau_{z_{E}}$, using DISASTER, the v0.1 version of DISENT and its fixed version. The event shape presented was calculated (at $x_{b j}=0.0039, Q^{2}=7.5 \mathrm{GeV}$ and $y=0.001$ ) with the programs Dispatch and DISresum, written by Salam et al. $[35,36,48]$. Similar results are obtained in the case of $\tau_{z_{Q}}$. We also found agreement between DISASTER and the modified version of DISENT for the quark channel, and for other event shapes. 


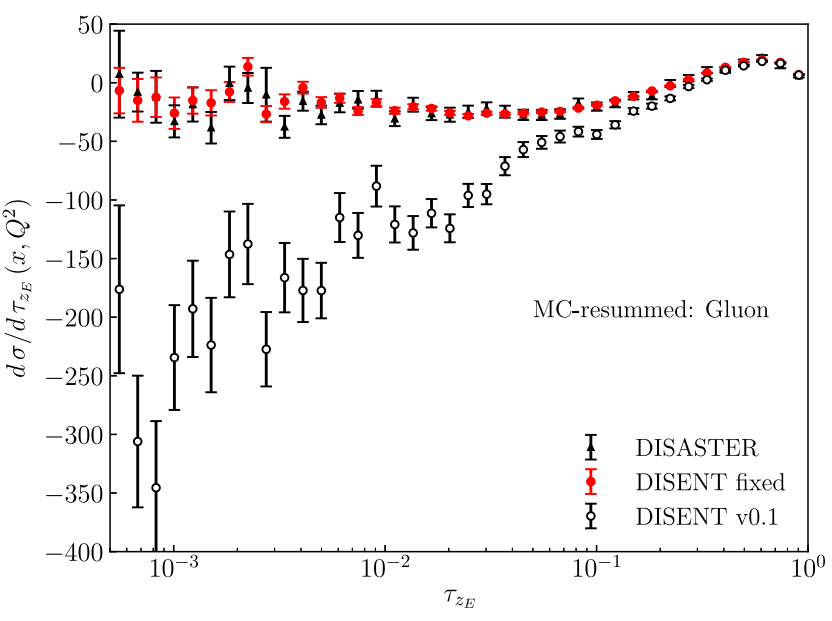

FIG. 16. Difference between the $\mathcal{O}\left(\alpha_{S}^{2}\right)$ coefficient for the fixed-order Monte Carlo calculation and the expansion of the resummed calculation for the gluonic contribution to $\tau_{z_{E}}$, using DISASTER, the original version of DISENT and its fixed version.

\section{APPENDIX B: SPIN CORRELATIONS}

As it was pointed out in Sec. III A, while, in principle, the initial-state dipole factorization formula for an $n$-parton scattering involves spin correlations between spindependent kernels and $(n-1)$-particle matrix elements, those correlations cancel in polarized cross sections. The appearance of such correlations, as well as their cancellation in the polarized case, are simpler to deduce within the helicity amplitudes formalism. Since the calculation of polarized cross sections involve differences between the helicity states of incoming particles, we consider an $n$-particle scattering of the form

$$
p_{a}^{\lambda_{a}}+p_{1}^{\lambda_{1}} \rightarrow p_{2}^{\lambda_{2}}+X_{f}^{\left\{\lambda_{X_{f}}\right\}},
$$

with $p_{1}$ and $p_{2}$ representing an incoming and an outgoing parton, respectively. The superscript $\lambda_{i}$ is used to indicate the helicity state of the $i$ th particle. $p_{a}$ represents an additional incoming particle, while $X_{f}$ is used for the remaining $n-3$ particles involved in the scattering. In the collinear limit between the partons 1 and 2, and following the notation from [49], the $n$-particle amplitude satisfies the strict factorization formula

$$
\begin{aligned}
\mathcal{M}^{n}\left(\lambda_{1}, \lambda_{2}, \lambda_{a},\left\{\lambda_{X_{f}}\right\}\right) \stackrel{1 \| 2}{\rightarrow} & g_{s} \sum_{c_{e}} \sum_{\lambda_{e}} \mathcal{C}\left(c_{e}, c_{1}, c_{2}\right) S_{1 e}^{\lambda_{1}, \lambda_{2}, \lambda_{e}}(z) \\
& \times \mathcal{M}^{n-1}\left(\lambda_{e}, \lambda_{a},\left\{\lambda_{X_{f}}\right\}\right),
\end{aligned}
$$

where $S_{1 e}^{\lambda_{1}, \lambda_{2}, \lambda_{e}}$ represents the splitting function with fixed helicities for the process $p_{1} \rightarrow p_{2}+p_{e}$, and $\mathcal{C}\left(c_{e}, c_{1}, c_{2}\right)$ is the associated color structure. Schematically, the collinear limit can be represented as in Fig. 17.

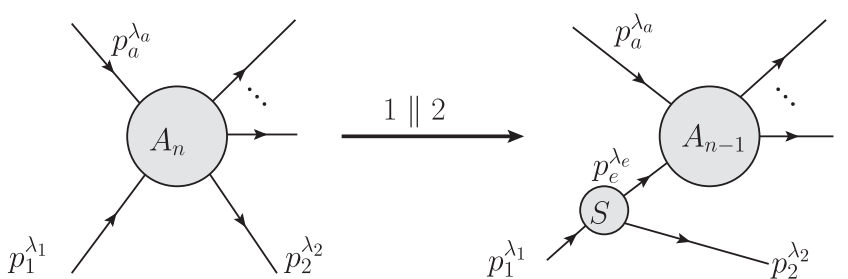

FIG. 17. Collinear behavior for the n-particle amplitude $\mathcal{M}^{n}$ in the limit $1 \| 2$.

It should be noted that, even after fixing all the external particles helicities, the factorization at the amplitude level involves the summation over the helicity states $\lambda_{e}$ of the intermediate parton. The case in which $p_{e}$ is a quark is trivial, since helicity conservation at the vertex implies that one of the terms in the sum over $\lambda_{e}$ is zero. The case with an intermediate gluon is, however, more involved. The exact factorization is lost at the squared-amplitude level, through the appearance of interference terms between the different helicities in the propagator. Those interference terms give rise to, precisely, the spin correlations noted in the dipole factorization formula. The exact form of the correlation terms can be easily obtained by squaring Eq. (B1):

$$
\begin{array}{r}
\left|\mathcal{M}^{n}\left(\lambda_{1}, \lambda_{2}, \lambda_{a},\left\{\lambda_{X_{f}}\right\}\right)\right|^{2} \stackrel{1 \| 2}{\rightarrow}\left|\mathcal{N}^{n}\left(\lambda_{1}, \lambda_{2}, \lambda_{a},\left\{\lambda_{X_{f}}\right\}\right)\right|^{2} \\
+\mathcal{R}\left(\lambda_{1}, \lambda_{2}, \lambda_{a},\left\{\lambda_{X_{f}}\right\}\right),
\end{array}
$$

where

$$
\begin{aligned}
\left|\mathcal{N}^{n}\left(\lambda_{1}, \lambda_{2}, \lambda_{a},\left\{\lambda_{X_{f}}\right\}\right)\right|^{2} \\
=g_{S}^{2} C\left|S_{1 g}^{\lambda_{1} \lambda_{2}+}(z)\right|^{2}\left|\mathcal{M}^{n-1}\left(+, \lambda_{a},\left\{\lambda_{X_{f}}\right\}\right)\right|^{2} \\
\quad+g_{S}^{2} C\left|S_{1 g}^{\lambda_{1} \lambda_{2}-}(z)\right|^{2}\left|\mathcal{M}^{n-1}\left(-, \lambda_{a},\left\{\lambda_{X_{f}}\right\}\right)\right|^{2},
\end{aligned}
$$

and the interference term is given by

$$
\begin{aligned}
\mathcal{R}\left(\lambda_{1}, \lambda_{2}, \lambda_{a},\left\{\lambda_{X_{f}}\right\}\right) & \\
= & 2 g_{S}^{2} C \operatorname{Re}\left\{S_{1 g}^{\lambda_{1} \lambda_{2}+}(z)\left(S_{1 g}^{\lambda_{1} \lambda_{2}-}(z)\right)^{*}\right. \\
& \left.\times \mathcal{M}^{n-1}\left(+, \lambda_{a},\left\{\lambda_{X_{f}}\right\}\right)\left(\mathcal{M}^{n-1}\left(-, \lambda_{a},\left\{\lambda_{X_{f}}\right\}\right)\right)^{*}\right\} .
\end{aligned}
$$

In Eqs. (B3) and (B4) we introduced the short-hand notation for the color factor

$$
C=\sum_{c_{1}, c_{2}, c_{e}, c_{e^{\prime}}} \frac{1}{N_{c_{1}}} \mathcal{C}\left(c_{e}, c_{1}, c_{2}\right) \mathcal{C}^{*}\left(c_{e^{\prime}}, c_{1}, c_{2}\right)
$$

with $1 / N_{c_{1}}$ denoting the average over the initial parton colors. For the relevant cases, and using the normalization from [50], $C$ can take the values $2 C_{A}$ and $C_{F}$, for an initial gluon and quark, respectively. Notice that the interference 
term depends on the initial parton helicity $\lambda_{1}$ only through the spin-dependent kernels $S_{1 g}^{\lambda_{1} \lambda_{2} \lambda_{e}}$. In the calculation of the unpolarized (polarized) cross section, we can then write:

$$
(\Delta) \sigma=\sum_{\lambda_{2}, \lambda_{a},\left\{\lambda_{X_{f}}\right\}} \frac{\left(\lambda_{a}\right)}{4} \times\left[\left|\mathcal{M}^{n}\left(+, \lambda_{2}, \lambda_{a},\left\{\lambda_{X_{f}}\right\}\right)\right|^{2}+(-)\left|\mathcal{M}^{n}\left(-, \lambda_{2}, \lambda_{a},\left\{\lambda_{X_{f}}\right\}\right)\right|^{2}\right],
$$

where the helicity factor $\left(\lambda_{a}\right)$ should only be considered in the polarized case. Using Eq. (B2), the unpolarized (polarized) cross section can in turn be expressed as

$$
\begin{aligned}
(\Delta) \sigma= & \sum_{\lambda_{2}, \lambda_{a},\left\{\lambda_{X_{f}}\right\}} \frac{\left(\lambda_{a}\right)}{4} \times\left[\left|\mathcal{N}^{n}\left(+, \lambda_{2}, \lambda_{a},\left\{\lambda_{X_{f}}\right\}\right)\right|^{2}+(-)\left|\mathcal{N}^{n}\left(-, \lambda_{2}, \lambda_{a},\left\{\lambda_{X_{f}}\right\}\right)\right|^{2}\right. \\
& \left.+\mathcal{R}\left(+, \lambda_{2}, \lambda_{a},\left\{\lambda_{X_{f}}\right\}\right)+(-) \mathcal{R}\left(-, \lambda_{2}, \lambda_{a},\left\{\lambda_{X_{f}}\right\}\right)\right] .
\end{aligned}
$$

We can then write explicitly the correlation terms in the second line of Eq. (B7) and sum over the polarizations of the final-state particles $X_{f}$

$$
\begin{aligned}
\sum_{\lambda_{2},\left\{\lambda_{X_{f}}\right\}} & {\left[\mathcal{R}\left(+, \lambda_{2}, \lambda_{a},\left\{\lambda_{X_{f}}\right\}\right)+(-) \mathcal{R}\left(-, \lambda_{2}, \lambda_{a},\left\{\lambda_{X_{f}}\right\}\right)\right] } \\
= & 2 g_{S}^{2} C \operatorname{Re}\left\{S_{1 g}^{+-+}(z)\left(S_{1 g}^{+--}(z)\right)^{*} \times \mathcal{M}^{n-1}\left(+, \lambda_{a}\right)\left(\mathcal{M}^{n-1}\left(-, \lambda_{a}\right)\right)^{*}\right\} \\
& +2 g_{S}^{2} C \operatorname{Re}\left\{S_{1 g}^{+++}(z)\left(S_{1 g}^{++-}(z)\right)^{*} \times \mathcal{M}^{n-1}\left(+, \lambda_{a}\right)\left(\mathcal{M}^{n-1}\left(-, \lambda_{a}\right)\right)^{*}\right\} \\
& +(-) 2 g_{S}^{2} C \operatorname{Re}\left\{S_{1 g}^{-++}(z)\left(S_{1 g}^{-+-}(z)\right)^{*} \times \mathcal{M}^{n-1}\left(+, \lambda_{a}\right)\left(\mathcal{M}^{n-1}\left(-, \lambda_{a}\right)\right)^{*}\right\} \\
& +(-) 2 g_{S}^{2} C \operatorname{Re}\left\{S_{1 g}^{--+}(z)\left(S_{1 g}^{---}(z)\right)^{*} \times \mathcal{M}^{n-1}\left(+, \lambda_{a}\right)\left(\mathcal{M}^{n-1}\left(-, \lambda_{a}\right)\right)^{*}\right\} \\
= & \left\{\begin{array}{lll}
4 g_{S}^{2} C \operatorname{Re}\left\{\left(S_{1 g}^{+-+}(z)\left(S_{1 g}^{+--}(z)\right)^{*}+S_{1 g}^{+++}(z)\left(S_{1 g}^{++-}(z)\right)^{*}\right) \times \mathcal{M}^{n-1}\left(+, \lambda_{a}\right)\left(\mathcal{M}^{n-1}\left(-, \lambda_{a}\right)\right)^{*}\right\} & \text { unpolarized } \\
0 & \text { polarized }
\end{array} .\right.
\end{aligned}
$$

In the last step we used that parity conservation implies that

$$
\begin{array}{ll}
S_{1 g}^{++-}(z)=\left(S_{1 g}^{--+}(z)\right)^{*}, & S_{1 g}^{+-+}(z)=\left(S_{1 g}^{-+-}(z)\right)^{*}, \\
S_{1 g}^{+++}(z)=\left(S_{1 g}^{---}(z)\right)^{*}, & S_{1 g}^{+--}(z)=\left(S_{1 g}^{-++}(z)\right)^{*},
\end{array}
$$

so all the interference terms in Eq. (B8) cancel each other in the polarized cross section. Thus, in the polarized case we simply obtain

$$
\begin{aligned}
\Delta \sigma= & \sum_{\lambda_{2}, \lambda_{a},\left\{\lambda_{X_{f}}\right\}} \frac{\lambda_{a}}{4}\left[\left|\mathcal{N}^{n}\left(+, \lambda_{2}, \lambda_{a},\left\{\lambda_{X_{f}}\right\}\right)\right|^{2}-\left|\mathcal{N}^{n}\left(-, \lambda_{2}, \lambda_{a},\left\{\lambda_{X_{f}}\right\}\right)\right|^{2}\right] \\
= & \sum_{\lambda_{2}, \lambda_{a},\left\{\lambda_{X_{f}}\right\}} \frac{\lambda_{a}}{4}\left[g_{S}^{2} C\left|S_{1 g}^{+\lambda_{2}+}(z)\right|^{2}\left|\mathcal{M}^{n-1}\left(+, \lambda_{a},\left\{\lambda_{X_{f}}\right\}\right)\right|^{2}+g_{S}^{2} C\left|S_{1 g}^{+\lambda_{2}-}(z)\right|^{2}\left|\mathcal{M}^{n-1}\left(-, \lambda_{a},\left\{\lambda_{X_{f}}\right\}\right)\right|^{2}\right. \\
& \left.-g_{S}^{2} C\left|S_{1 g}^{-\lambda_{2}+}(z)\right|^{2}\left|\mathcal{M}^{n-1}\left(+, \lambda_{a},\left\{\lambda_{X_{f}}\right\}\right)\right|^{2}-g_{S}^{2} C\left|S_{1 g}^{-\lambda_{2}-}(z)\right|^{2}\left|\mathcal{M}^{n-1}\left(-, \lambda_{a},\left\{\lambda_{X_{f}}\right\}\right)\right|^{2}\right] \\
= & \sum_{\left\{\lambda_{X_{f}}\right\}} \frac{4 \pi \alpha_{S}^{2}}{p_{1} \cdot p_{2}} \Delta P_{1 g}^{<}(z)\left|\Delta \mathcal{M}^{n-1}\left(\left\{\lambda_{X_{f}}\right\}\right)\right|^{2}=\frac{4 \pi \alpha_{S}^{2}}{p_{1} \cdot p_{2}} \Delta P_{1 g}^{<}(z)\left|\Delta \mathcal{M}^{n-1}\right|^{2},
\end{aligned}
$$

where we have used that the polarized Altarelli-Parisi kernels for $z<1, \Delta P_{1 j}^{<}(z)$, can be obtained from the helicitydependent kernels as

$$
\frac{1}{2 p_{i} \cdot p_{j}} \Delta P_{i j}^{<}(z)=\sum_{\lambda_{i}, \lambda_{j}} \lambda_{i} \lambda_{j}\left|S_{i j}^{\lambda_{i}+\lambda_{j}}(z)\right|^{2}=\sum_{\lambda_{i}, \lambda_{j}} \lambda_{i} \lambda_{j}\left|S_{i j}^{\lambda_{i}-\lambda_{j}}(z)\right|^{2}
$$

and defined 


$$
\left|\Delta \mathcal{M}^{n-1}\right|^{2} \equiv \sum_{\lambda_{e}, \lambda_{a},\left\{\lambda_{X_{f}}\right\}} \frac{\lambda_{e} \lambda_{a}}{4}\left|\mathcal{M}^{n-1}\left(\lambda_{e}, \lambda_{a},\left\{\lambda_{X_{f}}\right\}\right)\right|^{2}
$$

For the unpolarized cross section, a similar procedure leads to [49]:

$\sigma=\frac{4 \pi \alpha_{S}^{2}}{p_{1} \cdot p_{2}} P_{1 g}^{<}(z)\left|\mathcal{M}^{n-1}\right|^{2}+\frac{4 \pi \alpha_{S}^{2}}{p_{1} \cdot p_{2}} Q_{1 g}^{<}(z)\left|\tilde{\mathcal{M}}^{n-1}\right|^{2}$, where in the second term, the one that originates from spin correlations, we defined

$\left|\tilde{\mathcal{M}}^{n-1}\right|^{2}=\operatorname{Re}\left\{\frac{\langle 12\rangle}{[12]} \times \sum_{\lambda_{a}} \frac{1}{2} \mathcal{M}^{n-1}\left(+, \lambda_{a}\right)\left(\mathcal{M}^{n-1}\left(-, \lambda_{a}\right)\right)^{*}\right\}$,

while the factor $Q_{1 g}^{<}(z)$ takes the values $-4 C_{A} z(1-z)$ and $4 T_{R} z(1-z)$ for an initial gluon and quark, respectively.
[1] C. A. Aidala, S. D. Bass, D. Hasch, and G. K. Mallot, Rev. Mod. Phys. 85, 655 (2013).

[2] E. Aschenauer, A. Bazilevsky, K. Boyle, K. Eyser, R. Fatemi et al., arXiv:1304.0079.

[3] D. de Florian, R. Sassot, M. Stratmann, and W. Vogelsang, Phys. Rev. Lett. 113, 012001 (2014).

[4] D. de Florian, R. Sassot, M. Stratmann, and W. Vogelsang, Phys. Rev. D 80, 034030 (2009).

[5] E. R. Nocera, R. D. Ball, S. Forte, G. Ridolfi, and J. Rojo (NNPDF Collaboration), Nucl. Phys. B887, 276 (2014).

[6] A. Accardi et al., Eur. Phys. J. A 52, 268 (2016).

[7] E. C. Aschenauer, R. Sassot, and M. Stratmann, Phys. Rev. D 86, 054020 (2012).

[8] E. C. Aschenauer, R. Sassot, and M. Stratmann, Phys. Rev. D 92, 094030 (2015).

[9] E. C. Aschenauer, I. Borsa, G. Lucero, A. S. Nunes, and R. Sassot, Phys. Rev. D 102, 094018 (2020).

[10] V. Ravindran, J. Smith, and W. van Neerven, Nucl. Phys. B682, 421 (2004).

[11] E. Zijlstra and W. van Neerven, Nucl. Phys. B417, 61 (1994); B426, 245(E) (1994); B773, 105(E) (2007); B501, 599 (1997).

[12] A. Vogt, S. Moch, M. Rogal, and J. Vermaseren, Nucl. Phys. B, Proc. Suppl. 183, 155 (2008).

[13] S. Moch, J. Vermaseren, and A. Vogt, Nucl. Phys. B889, 351 (2014).

[14] S. Moch, J. Vermaseren, and A. Vogt, Phys. Lett. B 748, 432 (2015).

[15] M. Arratia, Y. Furletova, T. Hobbs, F. Olness, and S. J. Sekula, arXiv:2006.12520.

[16] Z.-B. Kang, X. Liu, S. Mantry, and D. Y. Shao, Phys. Rev. Lett. 125, 242003 (2020).

[17] S. Catani and M. Seymour, Nucl. Phys. B485, 291 (1997); B510, 503(E) (1998).

[18] I. Borsa, D. de Florian, and I. Pedron, Phys. Rev. Lett. 125, 082001 (2020).

[19] M. Cacciari, F. A. Dreyer, A. Karlberg, G. P. Salam, and G. Zanderighi, Phys. Rev. Lett. 115, 082002 (2015); 120, 139901(E) (2018).

[20] The code is available upon request from the authors

[21] V. Andreev et al. (H1 Collaboration), Eur. Phys. J. C 75, 65 (2015).
[22] V. Andreev et al. (H1 Collaboration), Eur. Phys. J. C 77, 215 (2017).

[23] H. Abramowicz et al. (ZEUS Collaboration), Eur. Phys. J. C 70, 965 (2010).

[24] S. Catani and M. Grazzini, Phys. Rev. Lett. 98, 222002 (2007).

[25] A. Gehrmann-De Ridder, T. Gehrmann, and E. Glover, J. High Energy Phys. 09 (2005) 056.

[26] R. Boughezal, K. Melnikov, and F. Petriello, Phys. Rev. D 85, 034025 (2012).

[27] M. Czakon, Phys. Lett. B 693, 259 (2010).

[28] T. Binoth and G. Heinrich, Nucl. Phys. B693, 134 (2004).

[29] C. Anastasiou, K. Melnikov, and F. Petriello, Phys. Rev. D 69, 076010 (2004).

[30] G. Somogyi, Z. Trocsanyi, and V. Del Duca, J. High Energy Phys. 01 (2007) 070.

[31] I. W. Stewart, F. J. Tackmann, and W. J. Waalewijn, Phys. Rev. Lett. 105, 092002 (2010).

[32] T. Gehrmann, A. Huss, J. Niehues, A. Vogt, and D. Walker, Phys. Lett. B 792, 182 (2019).

[33] J. Currie, T. Gehrmann, A. Huss, and J. Niehues, J. High Energy Phys. 07 (2017) 018.

[34] J. Vermaseren, A. Vogt, and S. Moch, Nucl. Phys. B724, 3 (2005).

[35] V. Antonelli, M. Dasgupta, and G. P. Salam, J. High Energy Phys. 02 (2000) 001.

[36] M. Dasgupta and G. P. Salam, J. High Energy Phys. 08 (2002) 032.

[37] G. McCance, in Workshop on Monte Carlo Generators for HERA Physics (Plenary Starting Meeting) (1999), pp. 151-159, https://inspirehep.net/literature/511711.

[38] Z. Nagy and Z. Trocsanyi, Phys. Rev. Lett. 87, 082001 (2001).

[39] G. 't Hooft and M. Veltman, Nucl. Phys. B44, 189 (1972).

[40] P. Breitenlohner and D. Maison, Commun. Math. Phys. 52, 11 (1977).

[41] W. Vogelsang, Nucl. Phys. B475, 47 (1996).

[42] E. Zijlstra and W. van Neerven, Nucl. Phys. B383, 525 (1992).

[43] J. Butterworth et al., J. Phys. G 43, 023001 (2016).

[44] D. de Florian, G. A. Lucero, R. Sassot, M. Stratmann, and W. Vogelsang, Phys. Rev. D 100, 114027 (2019). 
[45] S. Catani and B. Webber, J. High Energy Phys. 10 (1997) 005.

[46] S. Frixione and G. Ridolfi, Nucl. Phys. B507, 315 (1997).

[47] D. Graudenz, arXiv:hep-ph/9710244.
[48] M. Dasgupta and G. Salam, Phys. Lett. B 512, 323 (2001).

[49] S. Frixione, Z. Kunszt, and A. Signer, Nucl. Phys. B467, 399 (1996).

[50] M. L. Mangano and S. J. Parke, Phys. Rep. 200, 301 (1991). 